See discussions, stats, and author profiles for this publication at: https://www.researchgate.net/publication/4321721

\title{
Design of Distributed Detection Schemes for Multiaccess Channels
}

Conference Paper in IEEE Transactions on Aerospace and Electronic Systems · April 2012

DOI: 10.1109/AUSCTW.2008.4460821 · Source: IEEE Xplore

CITATIONS

12

READS

67

2 authors, including:

2. Jamie Scott Evans
University of Melbourne

243 PUBLICATIONS 3,904 CITATIONS

SEE PROFILE 


\section{Design of Distributed Detection Schemes for} Multiaccess Channels

FENG LI, Student Member, IEEE

JAMIE S. EVANS, Member, IEEE

SUBHRAKANTI DEY, Senior Member, IEEE

University of Melbourne

\begin{abstract}
We propose a methodology for designing the local mapping rule for fully synchronized but energy-limited sensors in a distributed detection system, where sensors communicate with the fusion center over multiaccess channels. Using the proposed methodology, we come up with the modified detect-and-forward scheme and the modified amplify-and-forward scheme. The performance of the two schemes is analyzed. We show that optimizing the local mapping rule can lead to a larger error exponent under total power constraint.
\end{abstract}

Manuscript received January 11, 2010; revised July 14, 2010; released for publication May 23, 2011.

IEEE Log No. T-AES/48/2/943834.

Refereeing of this contribution was handled by S. Marano.

This work was supported by the Australian Research Council.

The results of this paper were presented in part at the Australian Communications Theory Workshop (AusCTW), Christchurch, New Zealand, January 2008 and the IEEE International Conference on Acoustics, Speech and Signal Processing (ICASSP), Las Vegas, NV, March 2008.

Authors' address: ARC Special Research Centre for Ultra-Broadband Information Networks (CUBIN), Department of Electrical and Electronic Engineering, University of Melbourne, Lv 5, Building 193, Parkville, Victoria 3010, Australia, E-mail: (fenli@unimelb.edu.au).

$\overline{0018-9251 / 12 / \$ 26.00 ~(C) ~} 2012$ IEEE

\section{INTRODUCTION}

Signal processing utilizing spatially distributed sensors has become an important technology with a wide range of applications, due to the relatively low cost and the strong capability of sensor nodes. The enhanced computational ability allows part of the signal processing task to be done at local sensors, which reduces communication bandwidth and increases reliability. Detection with geographically dispersed sensors has attracted considerable attention. The state of nature to be detected, for example, could be the presence of an enemy target or the leakage of poisonous gas.

In a typical distributed detection system, each sensor first makes an observation, which is dependent on the true state of nature, locally processes it, and then communicates the processing result to the fusion center. Such a procedure is governed by a local mapping rule which describes how the measurement obtained by a sensor is processed and mapped to a signal to be transmitted to the fusion center. This mapping, which would probably be lossy (in terms of the amount of information being preserved in the mapping), is a major difference between the distributed detection system and the centralized detection system, where it is assumed that all raw observations are available to the fusion center. Based on the received signals, the fusion center will make a decision on the true state of nature.

Most of the works mentioned in [1] and [2] have not taken into consideration some features and limitations of communication channels between sensors and the fusion center. In a real distributed detection system, sensors will have to communicate with the fusion center over unreliable channels, which will distort the signals transmitted by local sensors. The design of distributed detection systems turns out to be a challenging task when sensor nodes are subject to harsh resource constraints. Power constraints will normally be applied, for example, to prolong the life time of the distributed system where sensors are powered by small batteries. In addition, the operating bandwidth of the system is usually limited which calls for a bandwidth-efficient communication scheme between sensors and the fusion center. In this paper we are interested in the art of distributed system design when we are given an unreliable channel and limited power and bandwidth.

\section{A. Related Works}

Early studies on the impact of unreliable channels can be found in [3] and [4]. Given nonideal channels, the design of optimal fusion rules for distributed detection systems utilizing binary sensors has been investigated in [5]-[7]. The unreliable communication channel is integrated into the joint optimization of the decision fusion rule and the local sensor quantizer in [8]. The asymptotic performance of 
distributed detection in energy-limited systems as the total power available goes large is analyzed in [9]. The performance of the amplify-and-forward approach for Gaussian signal detection is examined under a system power constraint in [10]. References [11]-[13] consider the situation where a sensor network is subject to transmission rate constraints. A low-communication-rate scheme is proposed in [14] where only informative sensor observations are allowed to be reported to the fusion center. This scheme is further developed in [15], and [16] considers the setup where communication constraints are replaced with some more general cost functions. The unreliable channel and the deficiency in power and bandwidth resources will normally induce a limit on the performance. Also due to lossy mappings, the performance of a distributed detection system can never be better than that of a centralized detection system.

However, it has been shown recently that for a noisy channel we can still design an asymptotically optimal distributed detection scheme for sensors under individual power constraints (IPCs) in the sense that the scheme can achieve the same error exponent as a centralized system can. Such asymptotically optimal schemes are reported in [17]-[19]. The key element that contributes to the success of those schemes is the use of multiple access when the channel gain is a non-zero constant. In contrast, parallel access is often adopted in traditional distributed detection schemes. The impacts of multiple access and parallel access on the performance have been evaluated and compared in [17]-[19], where multiaccess schemes demonstrate superior performance against schemes with parallel access. For discrete or quantized observations, motivated by the method of types [20], Mergen, Naware, and Tong [17] as well as Liu and Sayeed [18] proposed the type-based multiple access (TBMA) scheme, where the information of types is transmitted to the fusion center through a noisy multiaccess channel. Unlike schemes with the assumption that sensors have knowledge of the statistics of their observations, the TBMA scheme is suitable for sensors that are oblivious of observation statistics [18]. In the TBMA scheme, the bandwidth required is proportional to the number of types. The performance of the type-based method with random access is studied in [21]. For continuous sensor observations, it has been shown in [18] that the fusion of analog transmitted local log-likelihood ratio (LLR) over a multiaccess channel is asymptotically optimal with finite channel noise variances for conditionally independent sensor observations. In [19] the author proposed the fusion of amplified sensor observations. In addition to the asymptotic optimality, these schemes provide high efficiency in bandwidth and good system scalability (since the required bandwidth is fixed and does not depend on the number of sensors incorporated in the system). Under total power constraint (TPC), the TBMA scheme and the scheme proposed in [18] can achieve exponentially decreasing error probabilities. However, it is not clear whether these schemes can still provide the largest error exponents among all the schemes.

The multiaccess scheme also found its application in distributed inference problems. The authors of [22] study the scaling law of distributed inference when identical local mapping rule is assumed. In [23] it is shown that the Cramer-Rao bound on the asymptotic estimation error can be achieved for the exponential family of distributions using the sufficient-statistic based multiple access (SSBMA).

\section{B. Our Contributions}

In this paper the design of distributed detection systems with limited power and a stringent bandwidth constraint, where only one time slot of a channel is shared by all the sensors to communicate with the fusion center, is considered. We propose a general approach to design sensors' local mapping rules for such a distributed detection system. Under IPC it is shown in [18] that it is asymptotically optimal for sensors to transmit LLR of their observations when these observations are conditionally independent. The motivation for us to consider different local mapping rules is that the asymptotically optimal local mapping rule under IPC is not guaranteed to provide either the largest error exponent under TPC or the smallest error probabilities for a finite number of sensors. It is worthwhile to examine the performance of different local mapping rules and to understand their advantages and drawbacks.

Following the proposed methodology, we consider two particular designs, namely the modified detect-and-forward (MDF) and modified amplify-and-forward (MAF) schemes, under the assumption of conditionally independent and identically distributed (IID) sensor observations. The MDF scheme is a scheme which fuses local hard decisions over the multiaccess channel. A recent work that considers the fusion of local decisions over multiaccess channel is [24] where a RAKE receiver is proposed to deal with unsynchronized sensors. In our work we focus on a relative simple model, where we assume that sensors are fully synchronized, and derive some important theoretical results, which should provide readers a better understanding of fusing local decisions over a multiaccess channel. The MAF scheme adopts the amplify-and-forward technique, which has been widely studied in the research of cooperative diversity [25] and source-channel matching theory [26].

We show that the optimal fusion rule of the MDF scheme can be reduced to a threshold test on the received signal. Notice that the optimal decision fusion rule at the fusion center can always 
be expressed as a threshold test on the likelihood ratio of the received signal. However, it is not true that such an optimal likelihood ratio test can always be reduced to a threshold test on the received signal even if the local mapping rule is designed such that the magnitude of the transmitted signal monotonically increases with the likelihood ratio of the sensor's observation. The performance of the MDF and MAF schemes is analyzed in terms of error probabilities and error exponents. The most difficult part is to obtain the error exponents of both schemes under TPC. Although TPC is considered in [18] and [19], neither of the papers calculates the error exponents of their proposed distributed detection schemes under TPC. They either provide a bound on the error probability or derive the error exponent associated with a suboptimal decision fusion rule. We derive the error exponent of the MAF scheme under TPC analytically. With the help of the large deviation principle (LDP) and the discovery that the optimal decision fusion rule can be reduced to a simple threshold test on the received signal, we are able to numerically calculate the error exponent of the MDF scheme under TPC. An algorithm is provided and its output will eventually converge to the actual error exponent. The performance of the two schemes is compared under different situations and the question of whether to detect or to amplify is answered by these comparisons. An important observation is that the MAF scheme, which is proven to be asymptotically optimal under IPC, may result in a smaller error exponent than the MDF scheme under certain circumstances when TPC is imposed. This result simply suggests that the asymptotically optimal scheme under IPC is not guaranteed to provide the largest error exponent under TPC.

Different from the traditional detect-and-forward and amplify-and-forward schemes, the modified schemes offers higher energy efficiency by introducing a bias term to the sensor's local mapping rule (traditionally, only amplitude scaling is performed to satisfy a given power constraint, see [18] and [19]). Such an approach is considered in a slightly different context in [27]. But the author doesn't make any detailed analysis of the impact of the bias term on performance improvement. In our work we show that including the bias term will not help to increase the error exponent when IPC is considered but will lead to an increment in the error exponent when TPC is considered.

The rest of the paper is organized as follows. The problem formulation, together with the proposed design methodology, is provided in the next section. The MDF and MAF schemes are introduced in Section III and Section IV. The asymptotic performance of the two schemes is analyzed in Section V. The comparison of different schemes can be found in Section VI and we conclude in Section VII.

\section{PROBLEM FORMULATION}

Consider a binary hypothesis testing problem where a fusion center needs to decide between the two hypotheses $\left(H_{0}\right.$ and $\left.H_{1}\right)$ with the help of $N$ distributed sensors. The prior probabilities of both hypotheses (denoted by $P_{0}$ and $P_{1}$, respectively) are assumed known and none of them is equal to zero. The observation obtained by the $i$ th sensor is given by

$$
v_{i}= \begin{cases}s+n_{i} & \text { when } H_{1} \text { is true } \\ n_{i} & \text { when } H_{0} \text { is true }\end{cases}
$$

where $s$ is a known constant (without loss of generality, we assume $s$ is positive). For simplicity we assume $\left[n_{1}, \ldots, n_{N}\right]^{T}$ follows a Gaussian distribution with mean zero and covariance matrix $\sigma_{s}^{2} \mathbf{I}\left(\sigma_{s}>0\right)$, where $\mathbf{I}$ is the identity matrix. When $v_{i}$ is available, the $i$ th sensor will report it to the fusion center by transmitting a modulated signal. Use $M_{i}\left(v_{i}\right)$, a function of observation $v_{i}$, to denote the baseband signal to be modulated onto the carrier. Such a mapping from observation $v_{i}$ to $M_{i}\left(v_{i}\right)$ is determined by the local mapping rule $M_{i}(\cdot)$.

Here, we make the same assumption as made in [17] and [18] that all the sensors are fully synchronized. The communication channel between local sensors and the fusion center is modeled as an additive white Gaussian noise (AWGN) channel and coherent demodulation is adopted by the receiver of the fusion center. Under these assumptions we are able to perform distributed beamforming, which could help us to alleviate the performance degradation caused by the noisy channel. Geometrically distributed sensors are not perfectly synchronized by their nature. However, there have been proposed several schemes to synchronize distributed sensors. For example, a master-slave synchronization scheme is proposed in [28], which requires very little interaction between sensors and the fusion center. For a detailed summary of recent progress on distributed transmit beamforming technologies and algorithms, readers are referred to [29]. It is also demonstrated in [19] that slight mismatch in phases will have little impact on the performance. The case where unsynchronized sensors communicate local decisions to the fusion center over a Rayleigh fading multiaccess channel is considered in our paper [30] and the energy-efficient local mapping rule is also derived. The lack of channel knowledge and the loss of synchronization will introduce a detection error floor. However, the multiaccess scheme can still offer better performance than the parallel access scheme, when a relatively small number of sensors is deployed and the sensor's transmission power is low [30].

In the single-slot multiaccess scheme considered in this paper, all the sensors are required to transmit simultaneously. The baseband signal $w$ received 


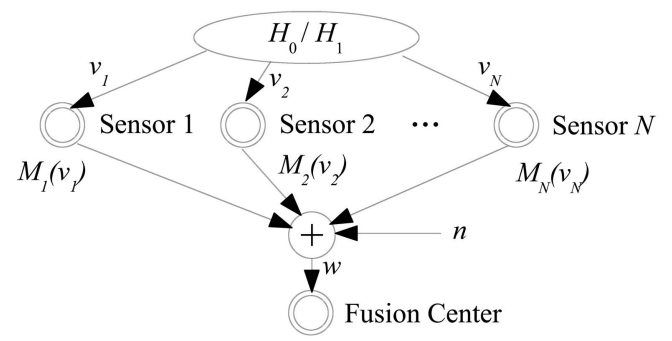

Fig. 1. System diagram.

by the fusion center will be a noisy version of the superposition of all $M_{i}\left(v_{i}\right)$, which can be expressed as

$$
w=\sum_{i=1}^{N} M_{i}\left(v_{i}\right)+n
$$

where $n$ is the additive Gaussian noise with mean zero and variance $\sigma_{c}^{2}$. This is illustrated in Fig. 1. Given $w$, a final decision $u(u=j \in\{0,1\}$ indicates that the fusion center decides $H_{j}$ ) will be made by the fusion center according to a certain fusion rule $\Gamma$. For the purpose of energy conservation, power constraints will be imposed. Both IPC and TPC are considered in this paper. IPC requires the following condition to be satisfied

$$
E\left[M_{i}\left(v_{i}\right)^{2}\right] \leq C_{I}, \quad i=1, \ldots, N
$$

where $C_{I}$ is the power budget for each individual sensor and $E[\cdot]$ denotes the expectation operation, while TPC demands

$$
\sum_{i=1}^{N} E\left[M_{i}\left(v_{i}\right)^{2}\right] \leq C_{T}
$$

where $C_{T}$ is the power budget for the whole system. Our purpose is to design local mapping rule $M_{i}(\cdot)$ which will provide low detection error probability under the given power constraint.

Some asymptotically optimal schemes may not be energy efficient (i.e., for any given $N$, we can find another scheme which is also asymptotically optimal but could achieve the same error probability with less power consumption). Moreover, asymptotically optimal schemes under IPC will not necessarily be optimal under TPC. This drives us to propose a general methodology to design and optimize local mapping rules for energy-limited sensors.

Suppose there exists a set of functions $\left\{f_{1}(\cdot), f_{2}(\cdot), \ldots, f_{N}(\cdot)\right\}$ such that $\Psi=\sum_{i=1}^{N} f_{i}\left(v_{i}\right)$ is a sufficient statistic for final decision making assuming that the fusion center can obtain each $f_{i}\left(v_{i}\right)$ without error. Here $f_{i}\left(v_{i}\right)$ could be viewed as a processed observation and can take either discrete or continuous value. Thus we can set $M_{i}\left(v_{i}\right)$ to be $\sqrt{\alpha} f_{i}\left(v_{i}\right)$ for all $i$, where $\alpha$ is a scaling factor to ensure that the power constraint (3) or (4) is satisfied. The received signal at the fusion center will be

$$
w=\sum_{i=1}^{N} \sqrt{\alpha} f_{i}\left(v_{i}\right)+n .
$$

Notice that for conditionally independent sensor observations, we can always find such a set of functions by setting $f_{i}(\cdot)$ to be the LLR of the observation $v_{i}$. This guarantees the best error exponent under IPC [18]. When the IPC is imposed, it is clear that the noise $n$ will be suppressed when we have a large number of sensors. Such a distributed beamforming may effectively suppress the noise at the receiver even under TPC. Consider a simple case where we have identical sensors (i.e., $f_{1}(\cdot)=\cdots=$ $\left.f_{N}(\cdot)=\tilde{f}(\cdot)\right)$. The maximum power that each sensor can get under TPC is $C_{T} / N$. Denote the second-order moment of $\tilde{f}\left(v_{i}\right)$ by $S$ (i.e., $E\left[\tilde{f}^{2}\left(v_{i}\right)\right]=S$ ). If all the sensors use the maximum power, we have $E\left[\alpha \tilde{f}^{2}\left(v_{i}\right)\right]=C_{T} / N$, which suggests that $\alpha=C_{T} / N S$. Now let's examine the power of $\sum_{i=1}^{N} \sqrt{\alpha} \tilde{f}\left(v_{i}\right)$. We have

$$
\begin{aligned}
& E\left[\left(\sum_{i=1}^{N} \sqrt{\alpha} \tilde{f}\left(v_{i}\right)\right)^{2}\right] \\
&=\sum_{i=1}^{N} \alpha E\left[\tilde{f}^{2}\left(v_{i}\right)\right]+2 \sum_{i=1}^{N} \sum_{j=i+1}^{N} \alpha E\left[\tilde{f}\left(v_{i}\right) \tilde{f}\left(v_{j}\right)\right] \\
&=C_{T}+2 \sum_{i=1}^{N} \sum_{j=i+1}^{N} \alpha E\left[\tilde{f}\left(v_{i}\right) \tilde{f}\left(v_{j}\right) \mid H_{0}\right] P_{0} \\
& \quad+2 \sum_{i=1}^{N} \sum_{j=i+1}^{N} \alpha E\left[\tilde{f}\left(v_{i}\right) \tilde{f}\left(v_{j}\right) \mid H_{1}\right] P_{1} \\
&=C_{T}+2 \sum_{i=1}^{N} \sum_{j=i+1}^{N} \alpha E\left[\tilde{f}\left(v_{i}\right) \mid H_{0}\right] E\left[\tilde{f}\left(v_{j}\right) \mid H_{0}\right] P_{0} \\
& \quad+2 \sum_{i=1}^{N} \sum_{j=i+1}^{N} \alpha E\left[\tilde{f}\left(v_{i}\right) \mid H_{1}\right] E\left[\tilde{f}\left(v_{j}\right) \mid H_{1}\right] P_{1} \\
&= C_{T}+\frac{C_{T}}{S}\left(E\left[\tilde{f}\left(v_{i}\right) \mid H_{0}\right]\right)^{2} P_{0}(N-1) \\
&\left.\left.\left.+v_{i}\right) \mid H_{1}\right]\right)^{2} P_{1}(N-1)
\end{aligned}
$$

As can be observed from (6), the power of $\sum_{i=1}^{N} \sqrt{\alpha} \tilde{f}\left(v_{i}\right)$ increases as the number of sensors increases.

We can further optimize the local mapping rule by setting $M_{i}\left(v_{i}\right)=\sqrt{\alpha}\left(f_{i}\left(v_{i}\right)-l\right)$ where $l$ is a bias. This will give us

$$
w=\sum_{i=1}^{N} \sqrt{\alpha}\left(f_{i}\left(v_{i}\right)-l\right)+n .
$$

For identical $f_{i}\left(v_{i}\right)$ considered in this paper, it can be proven that having all $M_{i}\left(v_{i}\right)$ adopt the same bias 
is actually optimal. For the more general case, we can set $M_{i}\left(v_{i}\right)=\sqrt{\alpha}\left(f_{i}\left(v_{i}\right)-l_{i}\right)$. We now compare (5) and (7). For any fixed $\alpha$, we will not degrade the performance by adopting (7) simply because the fusion center can add a constant amount $N \sqrt{\alpha} l$ to compensate for the bias effect. Since the power constraint depends on $M_{i}\left(v_{i}\right)$, we will be able to reduce the energy consumption by choosing a proper value for the bias. As a result, setting $f_{i}\left(v_{i}\right)$ to be the LLR of the $i$ th sensor's observation and making $l_{i}=0$ for all $i$ as in [18] will generally not be efficient in energy usage. To obtain the optimal value for the scaling factor and the bias, we have to find solutions for the following optimization problems

$$
\begin{aligned}
\min & P_{e} \\
\text { s.t. } & E\left[M_{i}\left(v_{i}\right)^{2}\right] \leq C_{I}, \quad i=1, \ldots, N
\end{aligned}
$$

and

$$
\begin{array}{ll}
\min & P_{e} \\
\text { s.t. } & \sum_{i=1}^{N} E\left[M_{i}\left(v_{i}\right)^{2}\right] \leq C_{T}
\end{array}
$$

where $P_{e}=\operatorname{Pr}\left\{u=1 \mid H_{0}\right\} \times P_{0}+\operatorname{Pr}\left\{u=0 \mid H_{1}\right\} \times P_{1}$ is the detection error probability. The analysis methods used in this paper can also be applied when our objective is to minimize some Bayes risk other than the error probability or when the Neyman-Pearson approach is adopted (rather than the Bayesian approach).

\section{MODIFIED DETECT AND FORWARD}

For identical local decision rules and conditionally IID sensor observations, it is well known that when 0/1 hard decisions are made at local sensors, the total number of positive decisions will be a sufficient statistic for final decision making [32]. Inspired by this result we propose the MDF scheme. First, a local decision $u_{i}=\gamma_{i}\left(v_{i}\right)$ (where $u_{i}=j \in\{0,1\}$ indicates that the local sensor decides $H_{j}$ and $\gamma_{i}(\cdot)$ is the local decision rule) will be made. From two possible signals, the $i$ th sensor then chooses the one corresponding to its decision $u_{i}$ and transmits it to the fusion center. The local mapping rule of the MDF scheme will have the following form

$$
M_{i}\left(v_{i}\right)=\left\{\begin{array}{lll}
\sqrt{\alpha}(1-l) & \text { when } & \gamma_{i}\left(v_{i}\right)=1 \\
\sqrt{\alpha}(-l) & \text { when } & \gamma_{i}\left(v_{i}\right)=0
\end{array} .\right.
$$

Finding optimal local decision rules is a difficult task even for parallel access schemes with reliable communication channels [1]. In our case it also turns out to be hard to obtain the optimal $\left\{\gamma_{1}(\cdot), \ldots, \gamma_{N}(\cdot)\right\}$. Here, for simplicity, we assume that sensors adopt the following common local decision rule

$$
\ln \frac{f\left(v_{i} \mid H_{1}\right)}{f\left(v_{i} \mid H_{0}\right)} \underset{H_{0}}{\stackrel{H_{1}}{\gtrless}} \ln \frac{P_{0}}{P_{1}}
$$

which means that the local sensor will decide $H_{1}$ to be true if the left-hand side of (11) is greater than its right-hand side and decide $H_{0}$ otherwise. When the decision rule (11) is used, the probability of local detection error $\operatorname{Pr}\left\{u_{i}=1 \mid H_{0}\right\} P_{0}+\operatorname{Pr}\left\{u_{i}=0 \mid H_{1}\right\} P_{1}$ will be minimized, and the detection probability and the false alarm probability at a local sensor are given by

$$
P_{d}=Q\left(\frac{2 \sigma_{s}^{2} \ln \frac{P_{0}}{P_{1}}-s^{2}}{2 s \sigma_{s}}\right)
$$

and

$$
P_{f}=Q\left(\frac{2 \sigma_{s}^{2} \ln \frac{P_{0}}{P_{1}}+s^{2}}{2 s \sigma_{s}}\right)
$$

respectively, where $Q(\cdot)$ is the complementary cumulative distribution function of a standard Gaussian. Since $Q(\cdot)$ is a decreasing function, we have

$$
0<P_{f}<P_{d}<1 .
$$

The conditional probability density functions of the signal received at the fusion center $w$ (see (2)), under $H_{0}$ and $H_{1}$ are given by

$$
\begin{aligned}
f\left(w \mid H_{0}\right)= & \sum_{i=0}^{N}\left(\begin{array}{c}
N \\
i
\end{array}\right) \frac{P_{f}^{i}\left(1-P_{f}\right)^{N-i}}{\sqrt{2 \pi \sigma_{c}^{2}}} \\
& \times \exp \left(-\frac{[w-(i \sqrt{\alpha}-N \sqrt{\alpha} l)]^{2}}{2 \sigma_{c}^{2}}\right)
\end{aligned}
$$

and

$$
\begin{aligned}
f\left(w \mid H_{1}\right)= & \sum_{i=0}^{N}\left(\begin{array}{c}
N \\
i
\end{array}\right) \frac{P_{d}^{i}\left(1-P_{d}\right)^{N-i}}{\sqrt{2 \pi \sigma_{c}^{2}}} \\
& \times \exp \left(-\frac{[w-(i \sqrt{\alpha}-N \sqrt{\alpha} l)]^{2}}{2 \sigma_{c}^{2}}\right) .
\end{aligned}
$$

At the fusion center the optimal fusion rule will be the following LLR test

$$
\ln \frac{f\left(w \mid H_{1}\right)}{f\left(w \mid H_{0}\right)} \underset{H_{0}}{\stackrel{H_{1}}{\gtrless}} \ln \frac{P_{0}}{P_{1}} .
$$

Define $\Omega_{1}$ to be the set in the domain of $w$ which contains all $w$ that will make the left-hand side of (17) greater than the right-hand side of (17) and $\Omega_{0}$ to be the complement set of $\Omega_{1} . \Omega_{0}$ and $\Omega_{1}$ are also known as the decision regions for $H_{0}$ and $H_{1}$, respectively. Due to the continuity of $f\left(w \mid H_{0}\right)$ and $f\left(w \mid H_{1}\right)$, the boundaries of $\Omega_{0}$ and $\Omega_{1}$ can be found by solving

$$
g(w)=f\left(w \mid H_{0}\right) P_{0}-f\left(w \mid H_{1}\right) P_{1}=0 .
$$


Substituting (15) and (16) into (18), we get

$$
\begin{aligned}
\sum_{i=0}^{N}\{ & {\left[P_{f}^{i}\left(1-P_{f}\right)^{N-i} P_{0}-P_{d}^{i}\left(1-P_{d}\right)^{N-i} P_{1}\right] } \\
& \left.\times\left(\begin{array}{c}
N \\
i
\end{array}\right) \exp \left(-\frac{(i \sqrt{\alpha})^{2}}{2 \sigma_{c}^{2}}\right) x^{i}\right\}=0
\end{aligned}
$$

where

$$
x=\exp \left(\frac{\sqrt{\alpha}(w+N \sqrt{\alpha} l)}{\sigma_{c}^{2}}\right) .
$$

Before exploring the optimal fusion rule, we provide one theorem.

LEMMA 1 Let $A_{0}$ and $A_{1}$ be two positive real numbers, $K$ be a positive integer, $0<p<1$ and $0<q<$ 1. The sequence $S_{k}=A_{0} p^{k}(1-p)^{K-k}-A_{1} q^{k}(1-q)^{K-k}$ with $k=0,1, \ldots, K$ will change its sign at most once.

PROOF The proof can be found in Appendix I.

THEOREM 1 The optimal fusion rule will be a threshold test on $w$.

PROOF Since the combination and the exponential function are always positive, the coefficients of $x^{i}$ on the left-hand side of (19) will change sign at most once according to Lemma 1. If the sign doesn't change, the fusion center will either always decide $H_{0}$ or always decide $H_{1}$. So the threshold of the test will be either $\infty$ or $-\infty$. If the sign of the coefficients changes once, (19) will have one positive root. To see this, we need the Descartes' rule of signs [33], which states that a real polynomial with $N_{v}$ variations in the signs of its coefficients has $N_{v}-2 k$ positive roots where $k$ is a nonnegative integer. Since $N_{v}$ in this case equals one, $k$ can only take the value of zero. It follows that there exists only one positive root and the left-hand side of (19) will change sign at this unique root. Since $x$ is a strictly increasing function of $w$ as shown by (20), (18) will also have one root and the sign of the left-hand side of (18) will change at this root. Thus (17) can be reduced to a threshold test on $w$.

It is worth noting that even if the local mapping rule $M_{i}(\cdot)$ is designed such that for arbitrary $v$ and $\tilde{v}$ we have $M_{i}(v)<M_{i}(\tilde{v})$ if $f\left(v \mid H_{1}\right) / f\left(v \mid H_{0}\right)<f\left(\tilde{v} \mid H_{1}\right) /$ $f\left(\tilde{v} \mid H_{0}\right)$, the optimal fusion rule cannot always be reduced to a threshold test on the received signal.

When the sign of the coefficients of $x^{i}$ in (19) changes once, (19) has a unique positive root $x_{r}$. The threshold $T$ for the test on $w$ can then be derived from (20) and is given by

$$
T=\frac{\sigma_{c}^{2} \ln x_{r}}{\sqrt{\alpha}}-N \sqrt{\alpha} l .
$$

Since $P_{d}>P_{f}$ and the sign of the coefficients of $x^{i}$ only changes once, we must have $P_{f}^{N} P_{0}-P_{d}^{N} P_{1}<0$.
Then for all sufficiently large $x$, the sign of the polynomial on the left-hand side of (19) will be negative. Therefore, $g(w)<0$ for all sufficiently large $w$ and the optimal fusion rule can be written as

$$
w \underset{H_{0}}{\stackrel{H_{1}}{\gtrless}} T \text {. }
$$

For test (22), the error probability can be expressed as

$$
\begin{aligned}
& P_{e}=\left(1-P_{D}\right) P_{1}+P_{F} P_{0} \\
&=\sum_{i=0}^{N}\left\{\left[P_{f}^{i}\left(1-P_{f}\right)^{N-i} P_{0}-P_{d}^{i}\left(1-P_{d}\right)^{N-i} P_{1}\right]\left(\begin{array}{c}
N \\
i
\end{array}\right)\right. \\
&\left.\quad \times Q\left(\frac{T-(i \sqrt{\alpha}-N \sqrt{\alpha} l)}{\sigma_{c}}\right)\right\}+P_{1}
\end{aligned}
$$

where $P_{D}$ and $P_{F}$ denote, respectively, the detection probability and the false alarm probability of the fusion center. We now establish the monotonic property of $P_{e}$.

THEOREM 2 Let $\eta=\sqrt{\alpha} / \sigma_{c}, P_{e}$ is a monotonically decreasing function of $\eta$.

PROOF The proof can be found in Appendix II.

Following from Theorem 2, (8) is equivalent to

$$
\begin{aligned}
\max & \alpha \\
\text { s.t. } & E\left[M_{i}\left(v_{i}\right)^{2}\right] \leq C_{I}, \quad i=1, \ldots, N .
\end{aligned}
$$

The constraints can be written as

$$
\begin{aligned}
C_{I} & \geq E\left[M_{i}\left(v_{i}\right)^{2}\right] \\
& =\left(P_{d} P_{1}+P_{f} P_{0}\right) \alpha(1-l)^{2}+\left(1-P_{d} P_{1}-P_{f} P_{0}\right) \alpha(-l)^{2} \\
& =\alpha\left[\left(l-P_{a}\right)^{2}+P_{a}-P_{a}^{2}\right]
\end{aligned}
$$

where $P_{a}=P_{d} P_{1}+P_{f} P_{0}$. By setting $l=P_{a}$, the maximum of $\alpha$ can be achieved. The optimal local mapping rule under IPC can be expressed as

$$
M_{i}\left(v_{i}\right)= \begin{cases}\sqrt{\alpha_{D I}^{*}}\left(1-P_{a}\right) & \text { when } \ln \frac{f\left(v_{i} \mid H_{1}\right)}{f\left(v_{i} \mid H_{0}\right)}>\ln \frac{P_{0}}{P_{1}} \\ \sqrt{\alpha_{D I}^{*}}\left(-P_{a}\right) & \text { otherwise }\end{cases}
$$

where $\alpha_{D I}^{*}=C_{I} /\left(P_{a}-P_{a}^{2}\right)$. Under TPC, the optimal scaling factor will be $\alpha_{D T}^{*}=C_{T} / N\left(P_{a}-P_{a}^{2}\right)$.

Now we look at a special case where $N=1$ and we consider IPC (TPC is equivalent to IPC when there is only one sensor). If we substitute $f\left(w \mid H_{0}\right)$ and $f\left(w \mid H_{1}\right)$ with $N=1$ into (18), we get

$$
\begin{aligned}
& {\left[P_{d} P_{1}-P_{f} P_{0}\right] \exp \left(-\frac{\left[w-\sqrt{\alpha_{D I}^{*}}\left(1-P_{a}\right)\right]^{2}}{2 \sigma_{c}^{2}}\right)} \\
& \quad=\left[\left(1-P_{f}\right) P_{0}-\left(1-P_{d}\right) P_{1}\right] \exp \left(-\frac{\left[w+\sqrt{\alpha_{D I}^{*}} P_{a}\right]^{2}}{2 \sigma_{c}^{2}}\right) .
\end{aligned}
$$


We assume that a finite $T$ exists. This implies $P_{d} P_{1}>$ $P_{f} P_{0}$ and $\left(1-P_{f}\right) P_{0}>\left(1-P_{d}\right) P_{1}$. So we can take logarithm at both sides of (25) and get a closed-form expression for $T$

$$
T=\frac{\alpha_{D I}^{*}\left(1-2 P_{a}\right)+2 \sigma_{c}^{2} \ln \frac{\left(1-P_{f}\right) P_{0}-\left(1-P_{d}\right) P_{1}}{P_{d} P_{1}-P_{f} P_{0}}}{2 \sqrt{\alpha_{D I}^{*}}} .
$$

Hence, the closed-form expression for $P_{e}$ will be available for the case where $N=1$.

Consider another special case where $P_{0}=P_{1}=0.5$; we have $P_{d}=Q\left(-s / 2 \sigma_{s}\right)>0.5$ and $P_{f}=Q\left(s / 2 \sigma_{s}\right)<$ 0.5 . Since $Q\left(-s / 2 \sigma_{s}\right)=1-Q\left(s / 2 \sigma_{s}\right)$, we have

$$
P_{d}=1-P_{f} \text {. }
$$

Equation (26) suggests that $P_{a}=0.5$. When the optimal local mapping rule is used (the optimal local mapping rule under IPC is given by (24) and we can get the optimal local mapping rule under TPC by replacing $\alpha_{D I}^{*}$ with $\alpha_{D T}^{*}$ in (24)), we have the following lemma.

LEMMA 2 When $P_{0}=P_{1}=0.5$, the optimal fusion rule is

$$
w \stackrel{H_{1}}{\gtrless} 0 .
$$

ProOF The proof can be found in Appendix III.

\section{MODIFIED AMPLIFY AND FORWARD}

In a centralized detection system, where all the sensor observations are available to the fusion center, the optimal fusion rule corresponding to the observation model (1) is an LLR test

$$
\ln \frac{f\left(v_{1}, \ldots, v_{N} \mid H_{1}\right)}{f\left(v_{1}, \ldots, v_{N} \mid H_{0}\right)} \underset{H_{0}}{\stackrel{H_{1}}{\gtrless}} \ln \frac{P_{0}}{P_{1}} .
$$

The assumption of conditionally independent observations allows us to simplify (28) to

$$
\sum_{i=1}^{N} v_{i} \underset{H_{0}}{\stackrel{H_{1}}{\gtrless}} \frac{2 \sigma_{s}^{2} \ln \frac{P_{0}}{P_{1}}+N s^{2}}{2 s}
$$

which suggests that $\Psi=\sum_{i=1}^{N} v_{i}$ is a sufficient statistic for final decision making in this case.

Motivated by the above result, we propose the MAF scheme where the local mapping rule has the following form

$$
M_{i}\left(v_{i}\right)=\sqrt{\alpha}\left(v_{i}-l\right)
$$

where $\alpha$ and $l$ are to be optimized. Substituting (1) and (29) into (2), we have

$$
w=\left\{\begin{array}{ll}
\sum_{i=1}^{N} \sqrt{\alpha}\left[\left(s+n_{i}\right)-l\right]+n & \text { when } H_{1} \text { is true } \\
\sum_{i=1}^{N} \sqrt{\alpha}\left(n_{i}-l\right)+n & \text { when } H_{0} \text { is true }
\end{array} .\right.
$$

At the fusion center, the optimal fusion rule is the LLR test (17). According to (30), $w \backsim \mathcal{N}\left(-N \sqrt{\alpha} l, N \alpha \sigma_{s}^{2}+\sigma_{c}^{2}\right)$ under $H_{0}$ and $w \backsim \mathcal{N}\left(N \sqrt{\alpha}(s-l), N \alpha \sigma_{s}^{2}+\sigma_{c}^{2}\right)$ under $H_{1}$ where $\mathcal{N}\left(\mu, \sigma^{2}\right)$ represents a Gaussian distribution with mean $\mu$ and variance $\sigma^{2}$. Hence, (17) becomes

$$
-\frac{[w-N \sqrt{\alpha}(s-l)]^{2}}{2\left(N \alpha \sigma_{s}^{2}+\sigma_{c}^{2}\right)}+\frac{(w+N \sqrt{\alpha} l)^{2}}{2\left(N \alpha \sigma_{s}^{2}+\sigma_{c}^{2}\right)} \underset{H_{0}}{\gtrless} \ln \frac{P_{0}}{P_{1}} .
$$

We can simplify (31) to a threshold test on $w$ which has the form (22) with threshold

$$
T=\frac{2\left(N \alpha \sigma_{s}^{2}+\sigma_{c}^{2}\right) \ln \frac{P_{0}}{P_{1}}+N^{2} \alpha s^{2}-2 N^{2} \alpha s l}{2 N \sqrt{\alpha} s} .
$$

The error probability can now be written as

$$
\begin{aligned}
P_{e}= & Q\left(\frac{T+N \sqrt{\alpha} l}{\sqrt{N \alpha \sigma_{s}^{2}+\sigma_{c}^{2}}}\right) P_{0} \\
& +\left[1-Q\left(\frac{T-N \sqrt{\alpha}(s-l)}{\sqrt{N \alpha \sigma_{s}^{2}+\sigma_{c}^{2}}}\right)\right] P_{1}
\end{aligned}
$$

where $T$ is given by (32). We now establish the monotonic property for the error probability of the MAF scheme as given in (33).

LEMMA 3 Let $\zeta=\sqrt{\left(N \alpha \sigma_{s}^{2}+\sigma_{c}^{2}\right) / N^{2} \alpha s^{2}}, P_{e}$ is a monotonically increasing function of $\zeta$.

PROOF It is easy to show that $d P_{e} / d \zeta>0$ and the lemma follows.

Due to Lemma 3, the minimum of $P_{e}$ can be found by minimizing $\zeta$ with the constraint (3) when IPC is considered. Problem (8) is equivalent to

$$
\begin{array}{ll}
\min & \sqrt{\frac{N \alpha \sigma_{s}^{2}+\sigma_{c}^{2}}{N^{2} \alpha s^{2}}} \\
\text { s.t. } & E\left[M_{i}\left(v_{i}\right)^{2}\right] \leq C_{I}, \quad i=1, \ldots, N .
\end{array}
$$

A quick glance tells us that $\zeta$ is a monotonically decreasing function of $\alpha$ and (34) is thus equivalent to

$$
\begin{aligned}
\max & \alpha \\
\text { s.t. } & E\left[M_{i}\left(v_{i}\right)^{2}\right] \leq C_{I}, \quad i=1, \ldots, N .
\end{aligned}
$$

Since the power constraint can be written as

$$
\begin{aligned}
E\left[M_{i}\left(v_{i}\right)^{2}\right] & =P_{1} E\left[\alpha\left(s+n_{i}-l\right)^{2}\right]+P_{0} E\left[\alpha\left(n_{i}-l\right)^{2}\right] \\
& =\alpha\left[\left(l-P_{1} s\right)^{2}+P_{1} P_{0} s^{2}+\sigma_{s}^{2}\right]
\end{aligned}
$$

the maximum of $\alpha$ is achieved by setting $l=P_{1} s$ and the optimized local mapping rule under IPC is given by

$$
M_{i}\left(v_{i}\right)=\sqrt{\alpha_{A I}^{*}}\left(v_{i}-P_{1} s\right)
$$

where $\alpha_{A I}^{*}=C_{I} /\left(\sigma_{s}^{2}+P_{1} P_{0} s^{2}\right)$. Under TPC, the optimal scaling factor will be $\alpha_{A T}^{*}=C_{T} / N\left(\sigma_{s}^{2}+P_{1} P_{0} s^{2}\right)$. Note 


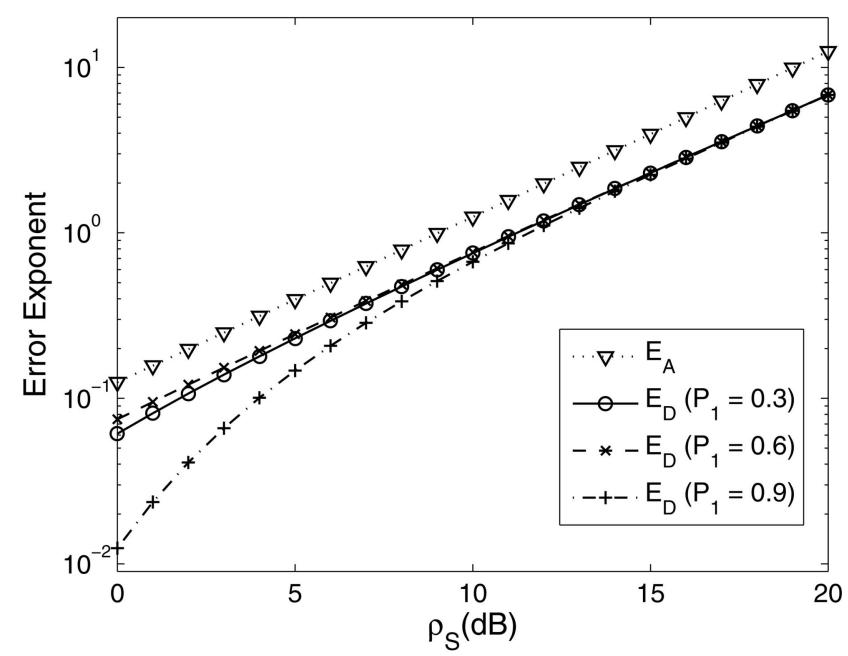

Fig. 2. Comparison of error exponents under IPC.

that the traditional amplify-and-forward scheme with $l=0$ is by no means optimal, because the optimal value for bias $l$ will never be 0 if $P_{1} \neq 0$.

\section{ASYMPTOTIC PERFORMANCE}

In this section we examine the asymptotic behaviors of both schemes under IPC and TPC. We define the signal-to-noise ratio (SNR) at the local sensor to be $\rho_{S}=s^{2} / \sigma_{s}^{2}$. We also define $\rho_{I}=C_{I} / \sigma_{c}^{2}$ which is the ratio of the power budget for each sensor to the channel noise power and $\rho_{T}=C_{T} / \sigma_{c}^{2}$ which is the ratio of the total power budget for the distributed system to the channel noise power.

\section{A. Under IPC}

We now calculate the error exponents of the MDF and MAF schemes under IPC. Although the optimal fusion rule of the MDF scheme turns out to be a threshold test on $w$ for any given $N$, it is difficult to find the closed-form expression for the error probability when $N \neq 1$. But this does not prevent us from evaluating the asymptotic behavior of the MDF scheme and a theorem is provided below.

THEOREM 3 The error exponent $E_{D I}$ of the $M D F$ scheme under IPC is given by

$$
E_{D I}=-\ln \left[P_{f}\left(\frac{P_{d}}{P_{f}}\right)^{\lambda^{*}}+\left(1-P_{f}\right)\left(\frac{1-P_{d}}{1-P_{f}}\right)^{\lambda^{*}}\right]
$$

where

$$
\lambda^{*}=\frac{\ln \left[\left(1-P_{f}\right) \ln \frac{1-P_{f}}{1-P_{d}}\right]-\ln \left[P_{f} \ln \frac{P_{d}}{P_{f}}\right]}{\ln \frac{P_{d}\left(1-P_{f}\right)}{P_{f}\left(1-P_{d}\right)}} .
$$

PROOF The proof can be found in Appendix IV.
In Fig. 2 we plot the error exponents of the MDF scheme for different $P_{1}$ and $\rho_{S}$. In the low SNR region, the prior probability $P_{1}$ will have a strong impact on the error exponent of the MDF scheme. However, the influence of $P_{1}$ on $E_{D I}$ becomes smaller and smaller as $\rho_{S}$ increases and $E_{D I}$ increases almost linearly in the high SNR region.

Note that the error probability $P_{e, A I}$ of the MAF scheme under IPC can be obtained from (33) by setting $\alpha=\alpha_{A I}^{*}$. Since the closed-form expression for error probability of the MAF scheme is available, the error exponent $E_{A I}$ of the MAF scheme under IPC can be calculated directly from the definition $E_{A I}=$ $\lim _{N \rightarrow \infty}-(1 / N) \ln P_{e, A I}$ and we give the following theorem without proof.

THEOREM 4 The error exponent $E_{A I}$ of the MAF scheme under IPC is given by

$$
E_{A I}=\frac{s^{2}}{8 \sigma_{s}^{2}}=\frac{\rho_{S}}{8} .
$$

The error exponent of the MAF scheme increases linearly with the SNR at the local sensor as shown in (38).

\section{B. Under TPC}

We first analyze the asymptotic behavior of the MDF scheme under TPC. Let

$$
\tilde{w}=\frac{w}{\sqrt{N}}=\frac{1}{N}\left(\sum_{i=1}^{N} \sqrt{N} M_{i}\left(v_{i}\right)+\sqrt{N} n\right)
$$

where $M_{i}\left(v_{i}\right)$ is given by

$$
M_{i}\left(v_{i}\right)= \begin{cases}\sqrt{\alpha_{D T}^{*}}\left(1-P_{a}\right) & \text { when } \ln \frac{f\left(v_{i} \mid H_{1}\right)}{f\left(v_{i} \mid H_{0}\right)}>\ln \frac{P_{0}}{P_{1}} . \\ \sqrt{\alpha_{D T}^{*}}\left(-P_{a}\right) & \text { otherwise }\end{cases}
$$

Since $\tilde{w}$ is a linear transformation from $w$, the smallest error probability resulting from distinguishing the two hypotheses using $\tilde{w}$ will be the same as that using $w$. We then construct $N$ IID Gaussian random variables $\hat{n}_{i}(i=1, \ldots, N)$ with mean zero and variance $\sigma_{c}^{2}$. Since $\sqrt{N} n$ in (39) follows $\mathcal{N}\left(0, N \sigma_{c}^{2}\right)$, the distribution of $\sum_{i=1}^{N} \hat{n}_{i}$ will be the same as that of $\sqrt{N} n$. Define $\hat{w}=(1 / N) \sum_{i=1}^{N}\left(\sqrt{N} M_{i}\left(v_{i}\right)+\hat{n}_{i}\right)$. Notice that $\hat{w}$ will have the same conditional distributions as $\tilde{w}$ under both hypotheses. We treat $w / \sqrt{N}$ as a realization of $\hat{w}$. Recall that $\sqrt{\alpha_{D T}^{*}}=\sqrt{C_{T} / N\left(P_{a}-P_{a}^{2}\right)}$. The distributions of $\sqrt{N} M_{i}\left(v_{i}\right)$ under $H_{0}$ and $H_{1}$ are governed by

$$
\operatorname{Pr}\left\{\sqrt{N} M_{i}\left(v_{i}\right)=x \mid H_{0}\right\}= \begin{cases}P_{f} & x=\sqrt{\hat{\alpha}}\left(1-P_{a}\right) \\ 1-P_{f} & x=\sqrt{\hat{\alpha}}\left(-P_{a}\right)\end{cases}
$$

and

$$
\operatorname{Pr}\left\{\sqrt{N} M_{i}\left(v_{i}\right)=x \mid H_{1}\right\}= \begin{cases}P_{d} & x=\sqrt{\hat{\alpha}}\left(1-P_{a}\right) \\ 1-P_{d} & x=\sqrt{\hat{\alpha}}\left(-P_{a}\right)\end{cases}
$$


where $\hat{\alpha}=C_{T} /\left(P_{a}-P_{a}^{2}\right)$. Obviously, $\sqrt{N} M_{i}\left(v_{i}\right)+\hat{n}_{i}$ are conditionally IID By the strong law of large numbers, we have $\operatorname{Pr}\left\{\lim _{N \rightarrow \infty} \hat{w}=\sqrt{\hat{\alpha}}\left(P_{f}-P_{a}\right) \mid H_{0}\right\}=1$ and $\operatorname{Pr}\left\{\lim _{N \rightarrow \infty} \hat{w}=\sqrt{\hat{\alpha}}\left(P_{d}-P_{a}\right) \mid H_{1}\right\}=1$. Design the fusion rule $\hat{\Gamma}_{\tau}$ as

$$
\hat{w} \stackrel{H_{1}}{\gtrless} \tau
$$

where $\tau$ is a fixed constant rather than a function of $N$. Since (40) will not be optimal for all $N$, the error exponent associated with the optimal fusion rule

$$
\ln \frac{f\left(\hat{w} \mid H_{1}\right)}{f\left(\hat{w} \mid H_{0}\right)} \underset{H_{0}}{\stackrel{H_{1}}{\gtrless}} \ln \frac{P_{0}}{P_{1}}
$$

will be lower bounded by

$$
\max _{\tau \in\left(\sqrt{\hat{\alpha}}\left(P_{f}-P_{a}\right), \sqrt{\hat{\alpha}}\left(P_{d}-P_{a}\right)\right)} E_{\hat{\Gamma}_{\tau}}
$$

where $E_{\hat{\Gamma}_{\tau}}$ is the error exponent associated with the fusion rule $\hat{\Gamma}_{\tau}$. Use $\Lambda_{0}(\lambda)$ and $\Lambda_{1}(\lambda)$ to denote the logarithmic moment generating functions of $\sqrt{N} M_{1}\left(v_{1}\right)+\hat{n}_{1}$ under $H_{0}$ and $H_{1}$, respectively. We have

$$
\begin{aligned}
\Lambda_{0}(\lambda)=\ln E & {\left[\exp \left(\lambda\left(\sqrt{N} M_{1}\left(v_{1}\right)+\hat{n}_{1}\right)\right) \mid H_{0}\right] } \\
=\ln ( & P_{f} \exp \left(\sqrt{\hat{\alpha}}\left(1-P_{a}\right) \lambda+\frac{\sigma_{c}^{2} \lambda^{2}}{2}\right) \\
& \left.+\left(1-P_{f}\right) \exp \left(-\sqrt{\hat{\alpha}} P_{a} \lambda+\frac{\sigma_{c}^{2} \lambda^{2}}{2}\right)\right) .
\end{aligned}
$$

Similarly,

$$
\begin{aligned}
\Lambda_{1}(\lambda)=\ln E & {\left[\exp \left(\lambda\left(\sqrt{N} M_{1}\left(v_{1}\right)+\hat{n}_{1}\right)\right) \mid H_{1}\right] } \\
=\ln ( & P_{d} \exp \left(\sqrt{\hat{\alpha}}\left(1-P_{a}\right) \lambda+\frac{\sigma_{c}^{2} \lambda^{2}}{2}\right) \\
& \left.+\left(1-P_{d}\right) \exp \left(-\sqrt{\hat{\alpha}} P_{a} \lambda+\frac{\sigma_{c}^{2} \lambda^{2}}{2}\right)\right) .
\end{aligned}
$$

Use $P_{F, \hat{\Gamma}_{\tau}}$ and $P_{M, \hat{\Gamma}_{\tau}}$ to denote the false alarm probability and the miss probability. Use $E_{F, \hat{\Gamma}_{\tau}}$ and $E_{M, \hat{\Gamma}_{\tau}}$ to denote the exponents of the false alarm probability and the miss probability. Applying the LDP [31], we will have

$$
\begin{aligned}
E_{M, \hat{\Gamma}_{\tau}} & =\lim _{N \rightarrow \infty}-\frac{1}{N} \ln P_{M, \hat{\Gamma}_{\tau}} \\
& =\lim _{N \rightarrow \infty}-\frac{1}{N} \ln \operatorname{Pr}\left\{\hat{w} \leq \tau \mid H_{1}\right\} \\
& =\inf _{x \leq \tau} \Lambda_{1}^{*}(x) \\
& =\Lambda_{1}^{*}(\tau) \\
& =\sup _{\lambda \in \mathbb{R}}\left[\lambda \tau-\Lambda_{1}(\lambda)\right] \\
& =-\inf _{\lambda \in \mathbb{R}}\left[\Lambda_{1}(\lambda)-\lambda \tau\right]
\end{aligned}
$$

where $\Lambda_{1}^{*}(x)$ is the Fenchel-Legendre transform of $\Lambda_{1}(\lambda)$. The third equality is due to the Cramer's Theorem [31] and the continuity of $\Lambda_{1}^{*}(x)$, and the fourth equality comes from the fact that $\Lambda_{1}^{*}(x)$ is a nonincreasing function of $x$ when $x<\sqrt{\hat{\alpha}}\left(P_{d}-P_{a}\right)$ [31]. Similarly, we have

$$
\begin{aligned}
E_{F, \hat{\Gamma}_{\tau}} & =\Lambda_{0}^{*}(\tau) \\
& =\sup _{\lambda \in \mathbb{R}}\left[\lambda \tau-\Lambda_{0}(\lambda)\right] \\
& =-\inf _{\lambda \in \mathbb{R}}\left[\Lambda_{0}(\lambda)-\lambda \tau\right]
\end{aligned}
$$

where $\Lambda_{0}^{*}(x)$ is the Fenchel-Legendre transform of $\Lambda_{0}(\lambda)$. For a given threshold $\tau$, the corresponding error exponent $E_{\hat{\Gamma}_{\tau}}$ is determined by the minimum of $E_{F, \hat{\Gamma}_{\tau}}$ and $E_{M, \hat{\Gamma}_{\tau}}$. Hence, (41) becomes

$$
\max _{\tau \in\left(\sqrt{\hat{\alpha}}\left(P_{f}-P_{a}\right), \sqrt{\hat{\alpha}}\left(P_{d}-P_{a}\right)\right)} \min \left(E_{F, \hat{\Gamma}_{\tau}}, E_{M, \hat{\Gamma}_{\tau}}\right) .
$$

Notice that $\Lambda_{0}(\lambda)$ and $\Lambda_{1}(\lambda)$ are both convex functions of $\lambda$ and that $-\lambda \tau$ is a linear function of $\lambda$ for fixed $\tau$. Thus, $\Lambda_{0}(\lambda)-\lambda \tau$ and $\Lambda_{1}(\lambda)-$ $\lambda \tau$ are both convex functions of $\lambda$. So for any given $\tau, E_{F, \hat{\Gamma}_{\tau}}$ and $E_{M, \hat{\Gamma}_{\tau}}$ can be obtained by using standard convex optimization techniques. Since $\Lambda_{0}^{*}(\tau)$ is a nondecreasing function of $\tau$ and $\Lambda_{1}^{*}(\tau)$ is a nonincreasing function of $\tau$ when $\tau \in$ $\left(\sqrt{\hat{\alpha}}\left(P_{f}-P_{a}\right), \sqrt{\hat{\alpha}}\left(P_{d}-P_{a}\right)\right), \min \left(E_{F, \hat{\Gamma}_{\tau}}, E_{M, \hat{\Gamma}_{\tau}}\right)$ will be a quasiconcave function and the maximum will be achieved when

$$
E_{F, \hat{\Gamma}_{\tau}}=E_{M, \hat{\Gamma}_{\tau}} .
$$

As a result, the lower bound (41) can be calculated numerically by using a two-stage optimization algorithm. The first stage is solving the nonlinear equation (42) by using the bisection method. For each iteration in the first stage, we need to calculate $E_{F, \hat{\Gamma}_{\tau}}$ and $E_{M, \hat{\Gamma}_{\tau}}$ for a particular value of $\tau$ by solving two convex optimization problems which forms the second stage. We are able to show that the lower bound is tight.

THEOREM 5 The error exponent $E_{D T}$ of the $M D F$ scheme under TPC is equal to that of a fixed threshold scheme where the fixed threshold $\hat{\tau}$ makes $E_{F, \hat{\Gamma}_{\hat{\tau}}}=$ $E_{M, \hat{\Gamma}_{\hat{\tau}}}$.

ProOF The proof can be found in Appendix V.

The closed-form expression for the error probability $P_{e, A T}$ of the MAF scheme under TPC can be obtained by setting $\alpha=\alpha_{A T}^{*}$ in (33). We give the following theorem without proof.

THEOREM 6 The error exponent $E_{A T}$ of the MAF scheme under TPC is given by

$$
E_{A T}=\frac{\rho_{S}}{8\left[1+\frac{P_{1} P_{0} \rho_{S}+1}{\rho_{T}}\right]} .
$$


Under TPC, the error exponent of the MAF scheme will depend not only on the SNR at the local sensor but also on the prior probabilities of both hypotheses and $\rho_{T}$. Notice there is a close relationship between (38) and (43). Under IPC, as the number of sensors goes large, the total system power tends to infinity. If we take the limit as $\rho_{T}$ goes to infinity (thus $C_{T}$ goes to infinity for fixed $\sigma_{c}^{2}$ ), we have

$$
\lim _{\rho_{T} \rightarrow \infty} E_{A T}=\frac{\rho_{S}}{8}=E_{A I} .
$$

1) A Special Case: In the following, we consider the special case where $P_{0}=P_{1}=0.5$. For this special case we can technically prove that under TPC the error exponent of the MDF scheme is larger than that of the MAF scheme when we have high quality sensors (i.e., $\rho_{S}$ is large) and low transmission power (i.e., $\rho_{T}$ is small). Since it is already shown that $P_{a}=$ 0.5 for this special case, we have $\hat{\alpha}=4 C_{T}$. Lemma 2 suggests that a test (see (40)) on $\hat{w}$ with fixed threshold $\tau=0$ is indeed optimal. The error exponent $E_{D T}$, the exponent of the miss probability $E_{M, \hat{\Gamma}_{0}}$, and the exponent of the false alarm probability $E_{F, \hat{\Gamma}_{0}}$ are all equal to each other. Hence,

$$
\begin{aligned}
E_{D T}= & E_{F, \hat{\Gamma}_{0}} \\
=-\inf _{\lambda \in \mathbb{R}} \ln ( & P_{f} \exp \left(\sqrt{C_{T}} \lambda+\frac{\sigma_{c}^{2} \lambda^{2}}{2}\right) \\
& \left.\quad+\left(1-P_{f}\right) \exp \left(-\sqrt{C_{T}} \lambda+\frac{\sigma_{c}^{2} \lambda^{2}}{2}\right)\right) .
\end{aligned}
$$

It is hard to obtain a closed-form expression for $E_{D T}$. However, we can find a closed-form expression for the lower bound of $E_{D T}$. Notice that $\tilde{\lambda}=\sqrt{C_{T}} / \sigma_{c}^{2}$ is the minimizer of $\left(1-P_{f}\right) \exp \left(-\sqrt{C_{T}} \lambda+\sigma_{c}^{2} \lambda^{2} / 2\right)$. The lower bound of $E_{D T}$ is hence

$$
\begin{aligned}
L B_{D T}=-\ln ( & P_{f} \exp \left(\sqrt{C_{T}} \tilde{\lambda}+\frac{\sigma_{c}^{2} \tilde{\lambda}^{2}}{2}\right) \\
& \left.+\left(1-P_{f}\right) \exp \left(-\sqrt{C_{T}} \tilde{\lambda}+\frac{\sigma_{c}^{2} \tilde{\lambda}^{2}}{2}\right)\right) \\
=-\ln ( & Q\left(\frac{\sqrt{\rho_{S}}}{2}\right) \exp \left(\frac{3 \rho_{T}}{2}\right) \\
& \left.+Q\left(-\frac{\sqrt{\rho_{S}}}{2}\right) \exp \left(-\frac{\rho_{T}}{2}\right)\right)
\end{aligned}
$$

where we have utilized the fact $P_{f}=Q\left(s / 2 \sigma_{s}\right)$. For the MAF scheme, the error exponent is given by

$$
E_{A T}=\frac{\rho_{S} \rho_{T}}{8 \rho_{T}+2 \rho_{S}+8} .
$$

We are interested in comparing $\exp \left(-L B_{D T}\right)$ with $\exp \left(-E_{A T}\right)$ under the scenario where $\rho_{S}$ is large and
$\rho_{T}$ is small. Expanding $\exp \left(3 \rho_{T} / 2\right), \exp \left(-\rho_{T} / 2\right)$, and $\exp \left(-E_{A T}\right)$ around $\rho_{T}=0$, we have

$$
\begin{aligned}
\exp \left(-L B_{D T}\right)= & Q\left(\frac{\sqrt{\rho_{S}}}{2}\right)\left(1+\frac{3 \rho_{T}}{2}+o_{1}\left(\rho_{T}\right)\right) \\
& +Q\left(-\frac{\sqrt{\rho_{S}}}{2}\right)\left(1-\frac{\rho_{T}}{2}+o_{2}\left(\rho_{T}\right)\right) \\
= & 1+\left[2 Q\left(\frac{\sqrt{\rho_{S}}}{2}\right)-\frac{1}{2}\right] \rho_{T} \\
& +Q\left(\frac{\sqrt{\rho_{S}}}{2}\right) o_{1}\left(\rho_{T}\right)+Q\left(-\frac{\sqrt{\rho_{S}}}{2}\right) o_{2}\left(\rho_{T}\right)
\end{aligned}
$$

and

$$
\exp \left(-E_{A T}\right)=1-\frac{\rho_{S}}{2\left(\rho_{S}+4\right)} \rho_{T}+o_{3}\left(\rho_{T} ; \rho_{S}\right)
$$

where $o_{1}\left(\rho_{T}\right), o_{2}\left(\rho_{T}\right)$, and $o_{3}\left(\rho_{T} ; \rho_{S}\right)$ contain terms of $\rho_{T}$ of second and higher orders. Hence,

$$
\begin{aligned}
\exp (- & \left.L B_{D T}\right)-\exp \left(-E_{A T}\right) \\
= & {\left[2 Q\left(\frac{\sqrt{\rho_{S}}}{2}\right)-\frac{1}{2}+\frac{\rho_{S}}{2\left(\rho_{S}+4\right)}\right] \rho_{T} } \\
& +Q\left(\frac{\sqrt{\rho_{S}}}{2}\right) o_{1}\left(\rho_{T}\right) \\
& +Q\left(-\frac{\sqrt{\rho_{S}}}{2}\right) o_{2}\left(\rho_{T}\right)-o_{3}\left(\rho_{T} ; \rho_{S}\right) .
\end{aligned}
$$

If $\rho_{S}$ is chosen such that

$$
\begin{aligned}
2 Q\left(\frac{\sqrt{\rho_{S}}}{2}\right)-\frac{1}{2}+\frac{\rho_{S}}{2\left(\rho_{S}+4\right)} \\
\quad=2 Q\left(\frac{\sqrt{\rho_{S}}}{2}\right)-\frac{2}{\rho_{S}+4}<0
\end{aligned}
$$

then for small enough $\rho_{T}$ we have $\exp \left(-L B_{D T}\right)<$ $\exp \left(-E_{A T}\right)$, which implies $L B_{D T}>E_{A T}$. If the lower bound of $E_{D T}$ is greater then $E_{A T}$, then $E_{D T}$ must be greater than $E_{A T}$. We now establish the condition for (44) to hold. An upper bound for $Q(x)$ is given by $Q(x) \leq \frac{1}{2} \exp \left(-x^{2} / 2\right)$. Hence, we have $2 Q\left(\sqrt{\rho_{S}} / 2\right) \leq \exp \left(-\rho_{S} / 8\right)$. Since $\exp \left(-\rho_{S} / 8\right)$ decreases exponentially as $\rho_{S}$ increases, for large $\rho_{S}$ we must have $\exp \left(-\rho_{S} / 8\right)<2 /\left(\rho_{S}+4\right)$, and (44) holds.

\section{COMPARISONS}

In this section, the MDF and MAF schemes are compared under both IPC and TPC. We first consider the case where there is a finite number of sensors deployed and then the case where there is an infinite number of sensors. Instead of only comparing the MDF scheme with the MAF scheme, we would also like to include two other schemes into the comparison which are the detect-and-forward and amplify-and-forward schemes. The detect-and-forward scheme uses the local mapping rule (10) with $l=0.5$. The amplify-and-forward scheme is the traditional 


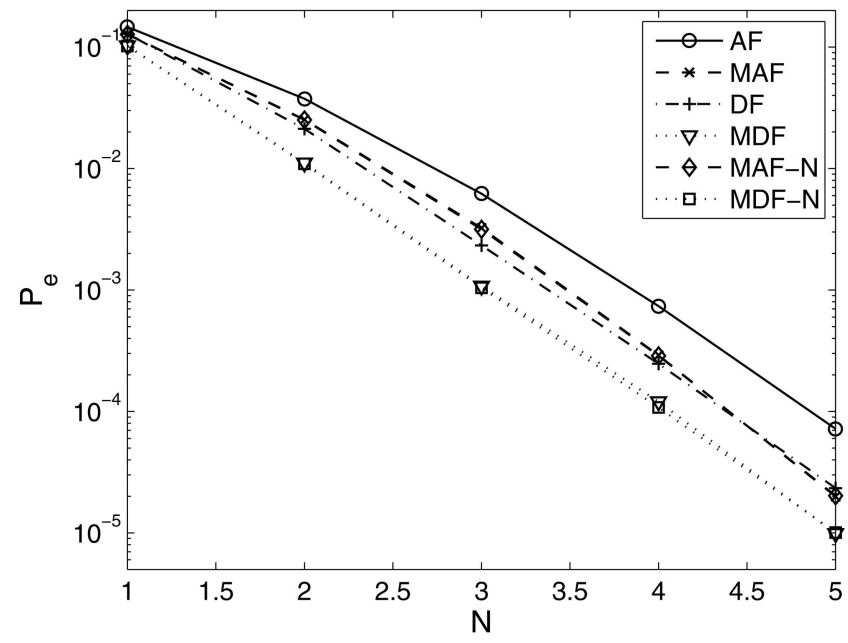

Fig. 3. Comparison of different schemes $\left(\rho_{S}=15 \mathrm{~dB}, \rho_{I}=0 \mathrm{~dB}\right.$, $\left.P_{1}=0.25\right)$.

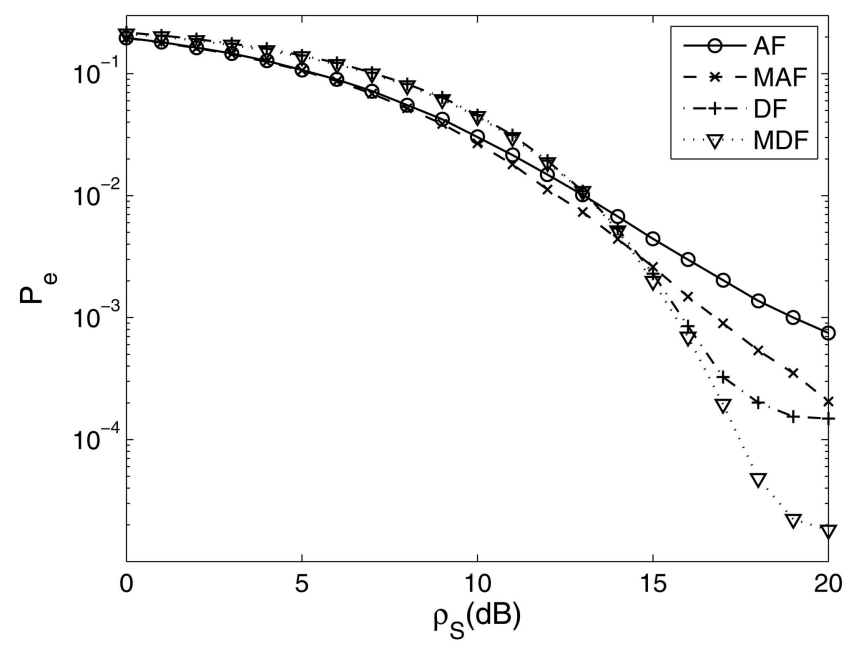

Fig. 4. Comparison of different schemes $\left(\rho_{I}=5 \mathrm{~dB}, P_{1}=0.25\right.$, $N=2)$.

amplify-and-forward scheme which uses the local mapping rule (29) with $l=0$. To make it a fair comparison, all the schemes are subject to the same energy constraint.

\section{A. Finite Number of Sensors}

Due to the symmetry of the problem, for any given $N$, TPC with power budget $C_{T}$ is equivalent to IPC with power budget $C_{I}=C_{T} / N$. As a result we only compare schemes under IPC for a finite number of sensors. For the two modified schemes, we plot both the simulation results and the numerically calculated error probabilities (using (23) and (33)) in Fig. 3. The simulation results agree very well with the theoretical results. It is shown in Fig. 3 that the error probabilities of all the four schemes decrease as the number of sensors increases. Since the local mapping rules of the MDF and MAF schemes are optimized for the given power budget, they are able to make less errors than their counterparts, which are the detect-and-forward

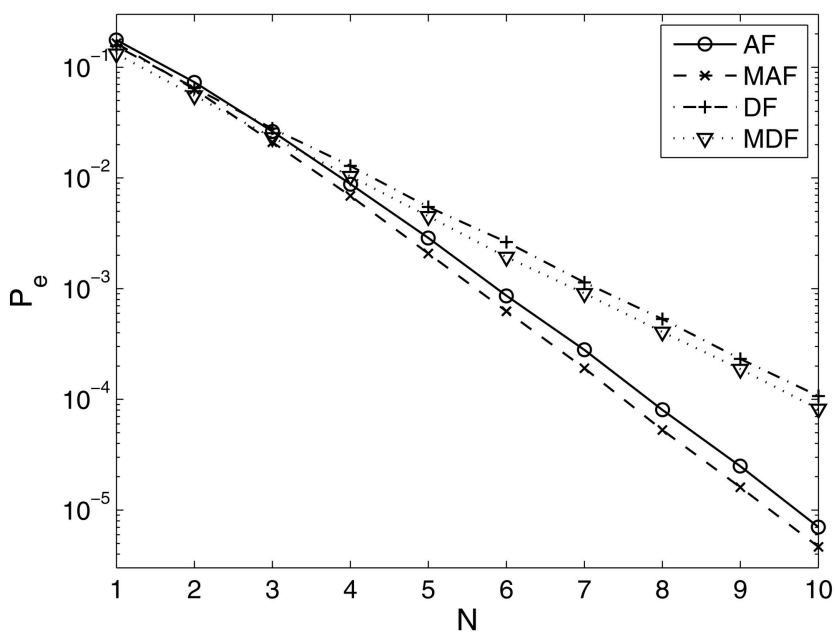

Fig. 5. Comparison of different schemes $\left(\rho_{S}=10 \mathrm{~dB}, \rho_{I}=0 \mathrm{~dB}\right.$, $\left.P_{1}=0.25\right)$.

and amplify-and-forward schemes, respectively. We can also observe that the MDF scheme outperforms the MAF scheme in this case. In Fig. 4 we keep the number of sensors and $\rho_{I}$ fixed and examine the impact of the quality of sensor observations on the detection performance. We find that the larger $\rho_{S}$ is, the smaller the error probability is. When $\rho_{S}$ is small, all the schemes will have similar performance. When $10 \mathrm{~dB}<\rho_{S}<20 \mathrm{~dB}$, the error probability of the MDF scheme decreases at the fastest rate as $\rho_{S}$ increases. This is because in the MDF scheme sensors can easily eliminate the noise contained in their observations by making local decisions when $\rho_{S}$ is large while sensors in the amplify-and-forward and MAF schemes will blindly forward the noise. This suggests that the MDF scheme will be a good choice when the quality of sensor observations is high.

\section{B. Asymptotic Behavior under IPC}

Notice that (38) does not depend on $\sigma_{c}^{2}$. When $\sigma_{c}^{2}=0$, the fusion center receives the noise-free shifted and scaled sum of observations (which is a sufficient statistic for a centralized system). It follows that the MAF scheme is asymptotically optimal under IPC.

Using similar approaches as used to establish Theorem 3 and Theorem 4, we can calculate the error exponents of the amplify-and-forward and detect-and-forward schemes. It turns out that the MAF and amplify-and-forward schemes have the same error exponents under IPC. So do the MDF and detect-and-forward schemes. This suggests that optimizing $\alpha$ and $l$ under IPC will not give us a gain in the error exponent.

As shown in Fig. 5 the error probabilities of all the schemes decrease exponentially when the number of sensors increases. Although the error probabilities of the amplify-and-forward and MAF schemes decrease at the same rate (i.e., the error exponents of the amplify-and-forward and MAF schemes 


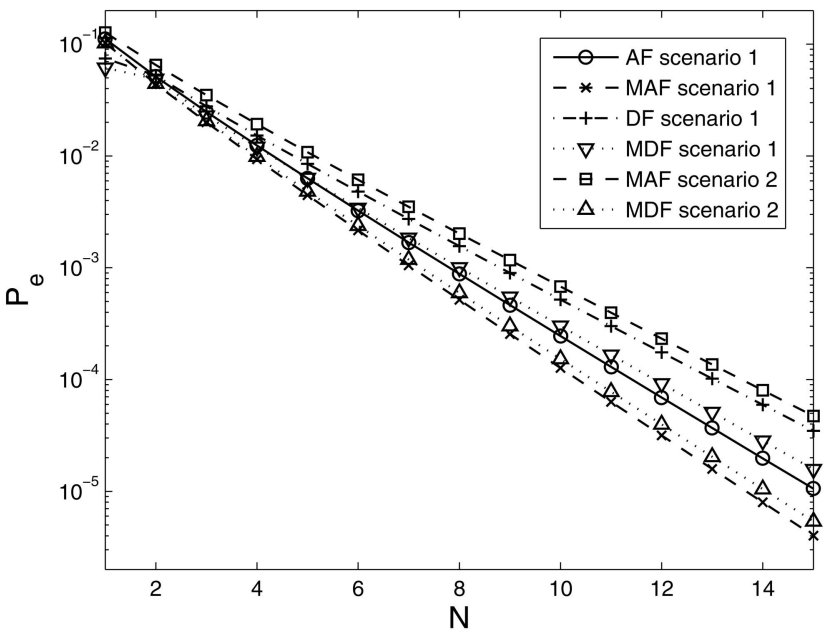

Fig. 6. Comparison of different schemes under TPC.

are the same), we can notice that there is always a performance gap between the amplify-and-forward and MAF schemes. Such a performance gap between the detect-and-forward and MDF schemes can also be found. Additionally, we can observe that the error probability of the MDF scheme decreases slower than that of the MAF scheme. This is because the MAF scheme is asymptotically optimal under IPC and thus has the largest error exponent. As a result, it will be good to adopt the MAF scheme when a large number of sensors with IPC are used.

\section{Asymptotic Behavior under TPC}

We can obtain the error exponent of the amplify-and-forward scheme under TPC using a similar approach as used to establish Theorem 6. We find that the error exponent of the amplify-and-forward scheme is smaller than that of the MAF scheme. Next, we look at the detect-and-forward and MDF schemes. Since the values for $\alpha$ and $l$ in the detect-and-forward scheme are different from those in the MDF scheme, the logarithmic moment generating functions of $\sqrt{N} M_{1}\left(v_{1}\right)+\hat{n}_{1}$ will be different for the two schemes. This will eventually lead to a difference in the error exponents of the two schemes. Since the MDF scheme is more energy efficient, it will have a larger error exponent than the detect-and-forward scheme. Different from the situations under IPC, we can achieve a gain in the error exponent by optimizing the local mapping rule under TPC.

We first investigate the scenario (called "scenario 1") where $\rho_{S}=10 \mathrm{~dB}, \rho_{T}=5 \mathrm{~dB}, P_{1}=0.25$. Fig. 6 shows the performance of the four schemes under TPC under scenario 1 . Thanks to the multiaccess strategy, the error probabilities of the four schemes still decrease exponentially even though the total energy of the system is kept constant. The MAF scheme has the largest error exponent compared with the other three schemes. We can also find

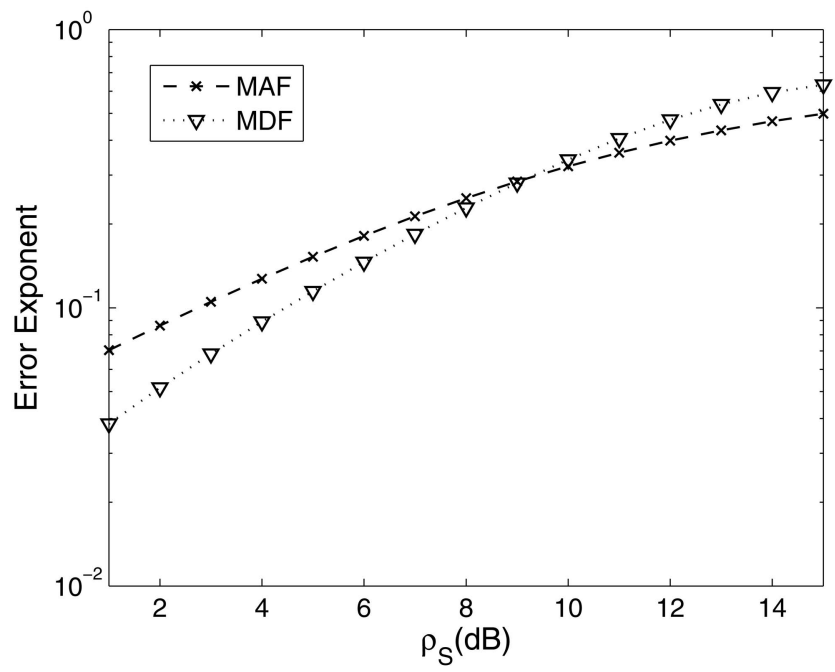

Fig. 7. Comparison of $\operatorname{MDF}$ and $\operatorname{MAF}\left(\rho_{T}=0 \mathrm{~dB}, P_{1}=0.25\right)$.

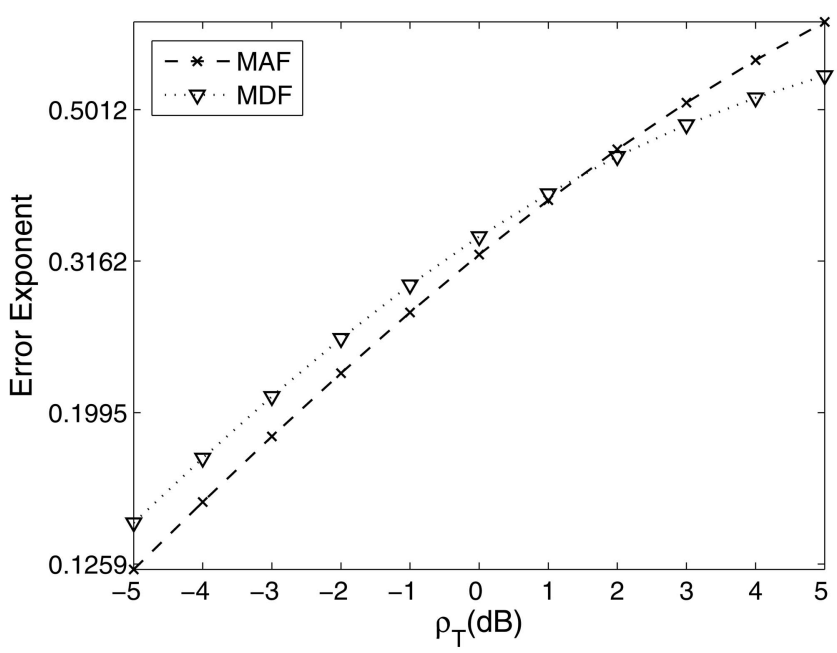

Fig. 8. Comparison of $\operatorname{MDF}$ and $\operatorname{MAF}\left(\rho_{S}=10 \mathrm{~dB}, P_{1}=0.25\right)$.

that the MDF scheme (MAF scheme) has larger error exponent than the detect-and-forward scheme (amplify-and-forward scheme respectively), which is different from Fig. 5 where the error probability of the MDF scheme (MAF scheme) decreases at the same rate as that of the detect-and-forward scheme (amplify-and-forward scheme respectively). The simulation results clearly show that we are able to achieve a larger error exponent under TPC by optimizing the local mapping rule. We then investigate the scenario (called "scenario 2") where $\rho_{S}=15 \mathrm{~dB}$, $\rho_{T}=0 \mathrm{~dB}, P_{1}=0.25$. By increasing $\rho_{S}$ by $5 \mathrm{~dB}$ and decreasing $\rho_{T}$ by $5 \mathrm{~dB}$, the MDF scheme beats the MAF schemes overwhelmingly as can be observed from Fig. 6.

In Fig. 7 and Fig. 8, we compare the error exponent of the MAF scheme with the error exponent of the MDF scheme fixing $\rho_{S}$ or $\rho_{T}$ and varying the value of the other parameter. We can observe that the MDF scheme is suitable for high quality sensors with low total transmission power even when 
a larger number of sensors are concerned. Notice that this observation has been technically proven in Section VB1 for the special case $P_{0}=P_{1}=0.5$.

\section{CONCLUSIONS}

We have considered the design of local mapping rules for an energy-limited distributed detection system. For a single-slot multiaccess channel, a design methodology is proposed which is based on the idea of communicating the noisy version of a sufficient statistic of processed sensor observations to the fusion center. Two energy-efficient schemes (the MDF and MAF schemes) are designed according to the proposed methodology. The optimal fusion rule of the MDF scheme is shown to be a simple threshold test on the received signal at the fusion center. Under IPC, the MAF scheme is asymptotically optimal while the MDF scheme is not. However, the MAF scheme is not guaranteed to be optimal for a finite number of sensors. Under TPC, the MDF scheme may have a larger error exponent than the MAF scheme when the system is composed of high quality sensors with a low total transmission power. By optimizing the local mapping rule, both the MDF and MAF schemes could offer larger error exponents than the unmodified schemes (i.e., the detect-and-forward and amplify-and-forward schemes respectively) under TPC.

\section{APPENDIX I. PROOF OF LEMMA 1}

Rewrite $S_{k}$ as $S_{k}=A_{0}(p /(1-p))^{k}(1-p)^{K}-$ $A_{1}(q /(1-q))^{k}(1-q)^{K}$. Without loss of generality, we assume $0<p<q<1$. We have

$$
\frac{q}{1-q}>\frac{p}{1-p} \text {. }
$$

Suppose for a given $\kappa$ we have $S_{\kappa}<0$. Then for all $k>\kappa$ we have $S_{k}<0$. This can be proved by induction. First, we examine $S_{\kappa+1} . S_{\kappa}<0$ implies that
$A_{1}(q /(1-q))^{\kappa}(1-q)^{K}>A_{0}(p /(1-p))^{\kappa}(1-p)^{K}$. Due to (45) we have

$$
\begin{aligned}
A_{1}\left(\frac{q}{1-q}\right)^{(\kappa+1)}(1-q)^{K} \\
=A_{1}\left(\frac{q}{1-q}\right)^{\kappa}(1-q)^{K}\left(\frac{q}{1-q}\right) \\
\quad>A_{0}\left(\frac{p}{1-p}\right)^{\kappa}(1-p)^{K}\left(\frac{q}{1-q}\right) \\
\quad>A_{0}\left(\frac{p}{1-p}\right)^{\kappa}(1-p)^{K}\left(\frac{p}{1-p}\right) \\
=A_{0}\left(\frac{p}{1-p}\right)^{(\kappa+1)}(1-p)^{K} .
\end{aligned}
$$

As a result $S_{\kappa+1}$ is also smaller than zero. Similarly, we can prove that $S_{\kappa+2}$ must be negative and so on. From the above results, we know that $S_{k}$ will always be nonnegative, or always negative, or first nonnegative and then negative. So the sign of $S_{k}$ will change at most once.

\section{APPENDIX II. PROOF OF THEOREM 2}

We prove the theorem by showing that the first derivative of $P_{e}$ with respect to $\eta$ is negative. Substituting (21) and $\eta=\sqrt{\alpha} / \sigma_{c}$ into (23), we have

$$
\begin{aligned}
P_{e}=\sum_{i=0}^{N}\{ & {\left[P_{f}^{i}\left(1-P_{f}\right)^{N-i} P_{0}-P_{d}^{i}\left(1-P_{d}\right)^{N-i} P_{1}\right] } \\
& \left.\times\left(\begin{array}{c}
N \\
i
\end{array}\right)\left[\frac{1}{2}-\frac{1}{2} \operatorname{erf}\left(\frac{\ln x_{r}}{\sqrt{2} \eta}-\frac{i \eta}{\sqrt{2}}\right)\right]\right\}+P_{1}
\end{aligned}
$$

where $\operatorname{erf}(\cdot)$ is the error function. Since the sign of the coefficients of $x^{i}$ in (19) changes once, there must be a $\kappa$ such that the coefficients of $x^{i}(i>\kappa)$ are all negative and the coefficients of $x^{i}(i \leq \kappa)$ are all nonnegative. We then have

$$
\begin{aligned}
\frac{\partial P_{e}}{\partial \eta}= & \sum_{i=0}^{N}\left\{\left[P_{f}^{i}\left(1-P_{f}\right)^{N-i} P_{0}-P_{d}^{i}\left(1-P_{d}\right)^{N-i} P_{1}\right]\left(\begin{array}{c}
N \\
i
\end{array}\right) \frac{-\exp \left(-\left(\frac{\ln x_{r}}{\sqrt{2} \eta}-\frac{i \eta}{\sqrt{2}}\right)^{2}\right)}{\sqrt{\pi}} \frac{d\left(\frac{\ln x_{r}}{\sqrt{2} \eta}-\frac{i \eta}{\sqrt{2}}\right)}{d \eta}\right\} \\
= & \sum_{i=0}^{N}\left\{\left[P_{f}^{i}\left(1-P_{f}\right)^{N-i} P_{0}-P_{d}^{i}\left(1-P_{d}\right)^{N-i} P_{1}\right]\left(\begin{array}{c}
N \\
i
\end{array}\right) \exp \left(-\frac{i^{2} \eta^{2}}{2}\right) \exp \left(i \ln x_{r}\right)\left[\frac{d\left(\frac{\ln x_{r}}{\sqrt{2} \eta}\right)}{d \eta}-\frac{\kappa}{\sqrt{2}}+\frac{\kappa-i}{\sqrt{2}}\right]\right\} \\
& \times \frac{-\exp \left(-\left(\frac{\ln x_{r}}{\sqrt{2} \eta}\right)^{2}\right)}{\sqrt{\pi}}
\end{aligned}
$$




$$
\begin{aligned}
= & \sum_{i=0}^{N}\left\{\left[P_{f}^{i}\left(1-P_{f}\right)^{N-i} P_{0}-P_{d}^{i}\left(1-P_{d}\right)^{N-i} P_{1}\right]\left(\begin{array}{c}
N \\
i
\end{array}\right) \exp \left(-\frac{i^{2} \eta^{2}}{2}\right) x_{r}^{i}\right\} \frac{-\exp \left(-\left(\frac{\ln x_{r}}{\sqrt{2} \eta}\right)^{2}\right)}{\sqrt{\pi}}\left(\frac{d\left(\frac{\ln x_{r}}{\sqrt{2} \eta}\right)}{d \eta}-\frac{\kappa}{\sqrt{2}}\right) \\
& +\sum_{i=0}^{N}\left\{\left[P_{f}^{i}\left(1-P_{f}\right)^{N-i} P_{0}-P_{d}^{i}\left(1-P_{d}\right)^{N-i} P_{1}\right]\left(\begin{array}{c}
N \\
i
\end{array}\right) \exp \left(-\frac{i^{2} \eta^{2}}{2}\right) x_{r}^{i} \frac{\kappa-i}{\sqrt{2}}\right\} \frac{-\exp \left(-\left(\frac{\ln x_{r}}{\sqrt{2} \eta}\right)^{2}\right)}{\sqrt{\pi}} \\
= & \sum_{i=0}^{N}\left\{\left[P_{f}^{i}\left(1-P_{f}\right)^{N-i} P_{0}-P_{d}^{i}\left(1-P_{d}\right)^{N-i} P_{1}\right]\left(\begin{array}{c}
N \\
i
\end{array}\right) \exp \left(-\frac{i^{2} \eta^{2}}{2}\right) x_{r}^{i} \frac{\kappa-i}{\sqrt{2}}\right\} \frac{-\exp \left(-\left(\frac{\ln x_{r}}{\sqrt{2} \eta}\right)^{2}\right)}{\sqrt{\pi}} .
\end{aligned}
$$

Notice that

$$
P_{f}^{i}\left(1-P_{f}\right)^{N-i} P_{0}-P_{d}^{i}\left(1-P_{d}\right)^{N-i} P_{1} \begin{cases}<0 & \text { when } \quad i>\kappa \\ \geq 0 & \text { when } \quad i \leq \kappa\end{cases}
$$

and

$$
\kappa-i\left\{\begin{array}{lll}
<0 & \text { when } \quad i>\kappa \\
\geq 0 & \text { when } \quad i \leq \kappa
\end{array} .\right.
$$

As a result, we have

$$
\frac{d P_{e}}{d \eta}<0
$$

\section{APPENDIX III. PROOF OF LEMMA 2}

When the optimal local mapping rule is used and $P_{d}=1-P_{f}$ (see (26)), the left-hand side of (19) becomes

$$
\begin{aligned}
\sum_{i=0}^{N}\{ & {\left[\left(1-P_{d}\right)^{i} P_{d}^{N-i} \frac{1}{2}-P_{d}^{i}\left(1-P_{d}\right)^{N-i} \frac{1}{2}\right]\left(\begin{array}{c}
N \\
i
\end{array}\right) } \\
& \left.\times \exp \left(-\frac{(i \sqrt{\alpha})^{2}}{2 \sigma_{c}^{2}}\right) \exp \left(\frac{i \sqrt{\alpha}\left(w+N \sqrt{\alpha} \frac{1}{2}\right)}{\sigma_{c}^{2}}\right)\right\}
\end{aligned}
$$

where $\alpha=\alpha_{D I}^{*}$ for IPC and $\alpha=\alpha_{D T}^{*}$ for TPC. Set $w=0$ and (46) becomes

$$
\begin{aligned}
& \frac{1}{2} \sum_{i=0}^{N}\left(1-P_{d}\right)^{i} P_{d}^{N-i}\left(\begin{array}{c}
N \\
i
\end{array}\right) \exp \left(-\frac{\alpha i(i-N)}{2 \sigma_{c}^{2}}\right) \\
& \quad-\frac{1}{2} \sum_{i=0}^{N} P_{d}^{i}\left(1-P_{d}\right)^{N-i}\left(\begin{array}{c}
N \\
i
\end{array}\right) \exp \left(-\frac{\alpha i(i-N)}{2 \sigma_{c}^{2}}\right) .
\end{aligned}
$$

Let $j=N-i$. The second part of (47) becomes

$$
\frac{1}{2} \sum_{j=0}^{N} P_{d}^{N-j}\left(1-P_{d}\right)^{j}\left(\begin{array}{c}
N \\
j
\end{array}\right) \exp \left(-\frac{\alpha(N-j)(-j)}{2 \sigma_{c}^{2}}\right)
$$

which is equal to the first part of (47). Hence $w=0$ is the root of (46) (or in other words, the root of $\left.f\left(w \mid H_{0}\right) P_{0}-f\left(w \mid H_{1}\right) P_{1}\right)$. Since $f\left(w \mid H_{0}\right) P_{0}-f\left(w \mid H_{1}\right) P_{1}$ has at most one root, $w=0$ must be the unique root. The sign of $f\left(w \mid H_{0}\right) P_{0}-f\left(w \mid H_{1}\right) P_{1}$ changes at this unique root. As a result, (27) is the optimal fusion rule.

\section{APPENDIX IV. PROOF OF THEOREM 3}

To prove this theorem, an upper bound for the error exponent will be derived first. If we assume that the fusion center can obtain each $M_{i}\left(v_{i}\right)$ without error, then the best achievable error exponent $E_{C}$ will be the Chernoff information [20]

$$
E_{C}=-\min _{0 \leq \lambda \leq 1} \ln E\left[\left(\frac{p_{1}\left(M_{1}\left(v_{1}\right)\right)}{p_{0}\left(M_{1}\left(v_{1}\right)\right)}\right)^{\lambda} \mid H_{0}\right]
$$

where $p_{0}(x)$ and $p_{1}(x)$ are probability mass functions of $M_{i}\left(v_{i}\right)$ under $H_{0}$ and $H_{1}$, and are given by

$$
p_{0}(x)= \begin{cases}P_{f} & x=\sqrt{\alpha_{D I}^{*}}\left(1-P_{a}\right) \\ 1-P_{f} & x=\sqrt{\alpha_{D I}^{*}}\left(-P_{a}\right)\end{cases}
$$

and

$$
p_{1}(x)=\left\{\begin{array}{ll}
P_{d} & x=\sqrt{\alpha_{D I}^{*}}\left(1-P_{a}\right) \\
1-P_{d} & x=\sqrt{\alpha_{D I}^{*}}\left(-P_{a}\right)
\end{array} .\right.
$$

Substituting (49) and (50) into (48), we get

$$
E_{C}=-\min _{0 \leq \lambda \leq 1} \ln \left[P_{f}\left(\frac{P_{d}}{P_{f}}\right)^{\lambda}+\left(1-P_{f}\right)\left(\frac{1-P_{d}}{1-P_{f}}\right)^{\lambda}\right] \text {. }
$$

Since

$$
h(\lambda)=P_{f}\left(\frac{P_{d}}{P_{f}}\right)^{\lambda}+\left(1-P_{f}\right)\left(\frac{1-P_{d}}{1-P_{f}}\right)^{\lambda}
$$

is a strictly convex function of $\lambda$ and $h(0)=h(1)>$ $h(1 / 2)$, the $\lambda$ that minimizes $h(\lambda)$ must be in the interval $(0,1)$. As a result, the constraint $0 \leq \lambda \leq 1$ can 
be removed and we have

$$
E_{C}=-\min \ln \left[P_{f}\left(\frac{P_{d}}{P_{f}}\right)^{\lambda}+\left(1-P_{f}\right)\left(\frac{1-P_{d}}{1-P_{f}}\right)^{\lambda}\right]
$$

The $\lambda^{*}$ that satisfies $d h(\lambda) / d \lambda=0$ is given by (37). So the upper bound for the error exponent of the MDF scheme is given by the right-hand side of (36).

For the local mapping rule (24), we design another fusion rule $\dot{\Gamma}$

$$
\frac{\ln \frac{P_{d}\left(1-P_{f}\right)}{P_{f}\left(1-P_{d}\right)}}{\sqrt{\alpha_{D I}^{*}} N} w \underset{H_{0}}{\gtrless}-\left(\ln \frac{P_{d}\left(1-P_{f}\right)}{P_{f}\left(1-P_{d}\right)}\right) P_{a}-\ln \frac{1-P_{d}}{1-P_{f}} .
$$

We now show that the error exponent of the system $\mathcal{S}$ with local mapping rule (24) and fusion rule (52) will reach the upper bound we derived before. Using the fact that

$$
\ln \frac{p_{1}\left(\sqrt{\alpha_{D I}^{*}}\left(1-P_{a}\right)\right)}{p_{0}\left(\sqrt{\alpha_{D I}^{*}}\left(1-P_{a}\right)\right)}=\ln \frac{P_{d}}{P_{f}}
$$

and

$$
\ln \frac{p_{1}\left(\sqrt{\alpha_{D I}^{*}}\left(-P_{a}\right)\right)}{p_{0}\left(\sqrt{\alpha_{D I}^{*}}\left(-P_{a}\right)\right)}=\ln \frac{1-P_{d}}{1-P_{f}}
$$

we can reorganize (52) and get

$$
\omega=\sum_{i=1}^{N} \ln \frac{p_{1}\left(M_{i}\left(v_{i}\right)\right)}{p_{0}\left(M_{i}\left(v_{i}\right)\right)}+\frac{\left(\ln \frac{P_{d}\left(1-P_{f}\right)}{P_{f}\left(1-P_{d}\right)}\right) n}{\sqrt{\alpha_{D I}^{*}}} \underset{H_{0}}{\gtrless} 0 .
$$

For any $\lambda>0$, we have

$$
\begin{aligned}
P_{F, \mathcal{S}} & =\operatorname{Pr}\left\{\omega>0 \mid H_{0}\right\} \\
& \leq E\left[\exp (\lambda \omega) \mid H_{0}\right] \\
& =\left\{E\left[\left(\frac{p_{1}\left(M_{1}\left(v_{1}\right)\right)}{p_{0}\left(M_{1}\left(v_{1}\right)\right)}\right)^{\lambda} \mid H_{0}\right]\right\}^{N} E\left[\exp (\lambda k n) \mid H_{0}\right]
\end{aligned}
$$

where

$$
k=\frac{\ln \frac{P_{d}\left(1-P_{f}\right)}{P_{f}\left(1-P_{d}\right)}}{\sqrt{\alpha_{D I}^{*}}}
$$

and $P_{F, \mathcal{S}}$ is the false alarm probability of system $\mathcal{S}$. The inequality comes from the Markov inequality and the last equality is because $M_{i}\left(v_{i}\right)$ are conditionally IID and $n$ is independent of $M_{i}\left(v_{i}\right)$. Since $\lambda^{*}>0$, we have

$$
\begin{gathered}
-\frac{1}{N} \ln P_{F, \mathcal{S}} \geq-\ln E\left[\left(\frac{p_{1}\left(M_{1}\left(v_{1}\right)\right)}{p_{0}\left(M_{1}\left(v_{1}\right)\right)}\right)^{\lambda^{*}} \mid H_{0}\right] \\
-\frac{1}{N} \ln E\left[\exp \left(\lambda^{*} k n\right) \mid H_{0}\right] .
\end{gathered}
$$

For any $\lambda<0$, we have

$$
\begin{aligned}
P_{M, \mathcal{S}} & =\operatorname{Pr}\left\{\omega \leq 0 \mid H_{1}\right\} \\
& \leq E\left[\exp (\lambda \omega) \mid H_{1}\right] \\
& =\left\{E\left[\left(\frac{p_{1}\left(M_{1}\left(v_{1}\right)\right)}{p_{0}\left(M_{1}\left(v_{1}\right)\right)}\right)^{\lambda} \mid H_{1}\right]\right\}^{N} E\left[\exp (\lambda k n) \mid H_{1}\right]
\end{aligned}
$$

where $P_{M, \mathcal{S}}$ is the miss probability of system $\mathcal{S}$. Since $\lambda^{*}-1<0$,

$$
\begin{aligned}
-\frac{1}{N} \ln P_{M, \mathcal{S}} \geq & -\ln E\left[\left(\frac{p_{1}\left(M_{1}\left(v_{1}\right)\right)}{p_{0}\left(M_{1}\left(v_{1}\right)\right)}\right)^{\lambda^{*}-1} \mid H_{1}\right] \\
& -\frac{1}{N} \ln E\left[\exp \left(\left(\lambda^{*}-1\right) k n\right) \mid H_{1}\right] \\
= & -\ln E\left[\left(\frac{p_{1}\left(M_{1}\left(v_{1}\right)\right)}{p_{0}\left(M_{1}\left(v_{1}\right)\right)}\right)^{\lambda^{*}} \mid H_{0}\right] \\
& -\frac{1}{N} \ln E\left[\exp \left(\left(\lambda^{*}-1\right) k n\right) \mid H_{1}\right] .
\end{aligned}
$$

Notice that

$$
-\ln E\left[\left(\frac{p_{1}\left(M_{1}\left(v_{1}\right)\right)}{p_{0}\left(M_{1}\left(v_{1}\right)\right)}\right)^{\lambda^{*}} \mid H_{0}\right]=E_{C}
$$

and

$$
\begin{array}{r}
\lim _{N \rightarrow \infty}-\frac{1}{N} \ln E\left[\exp \left(\lambda^{*} k n\right) \mid H_{0}\right]=0 \\
\lim _{N \rightarrow \infty}-\frac{1}{N} \ln E\left[\exp \left(\left(\lambda^{*}-1\right) k n\right) \mid H_{1}\right]=0 .
\end{array}
$$

We use $E_{\mathcal{S}}$ to denote the error exponent of system $\mathcal{S}$. Due to (53)-(57) and the trivial upper bound $E_{C} \geq E_{\mathcal{S}}$, we can obtain $E_{C}=E_{\mathcal{S}}$. Since the fusion rule (17) is optimal for any given $N$ while (52) is not, we will have $E_{D I} \geq E_{\mathcal{S}}$. Thus the error exponent of the MDF scheme will also be able to attain the upper bound $E_{C}$. The theorem follows.

\section{APPENDIX V. PROOF OF THEOREM 5}

Let $\hat{\tau}$ be such that $E_{F, \hat{\Gamma}_{\hat{\hat{\tau}}}}=E_{M, \hat{\Gamma}_{\hat{\tau}}}$. Threshold $\hat{\tau}$ yields miss probability $P_{M, \hat{\Gamma}_{\hat{\tau}}}$ and false alarm probability $P_{F, \hat{\Gamma}_{\hat{\tau}}}$. As shown in Theorem 1, for any given $N$ the optimal test is also a threshold test with optimal threshold $\tau_{N}^{*}$ and the resulting miss probability and false alarm probability are given by $P_{M}^{*}=$ $\operatorname{Pr}\left\{\hat{w} \leq \tau_{N}^{*} \mid H_{1}\right\}$ and $P_{F}^{*}=\operatorname{Pr}\left\{\hat{w}>\tau_{N}^{*} \mid H_{0}\right\}$, respectively. If $\tau_{N}^{*}>\hat{\tau}$, we will have $P_{M}^{*} \geq P_{M, \hat{\Gamma}_{\hat{\tau}}}=\operatorname{Pr}\left\{\hat{w} \leq \hat{\tau} \mid H_{1}\right\}$. If $\tau_{N}^{*}<\hat{\tau}$, we will have $P_{F}^{*} \geq P_{F, \hat{\Gamma}_{\hat{\tau}}}$. Hence, $P_{1} P_{M}^{*}+$ $P_{0} P_{F}^{*} \geq \min \left(P_{0}, P_{1}\right) \cdot \min \left(P_{M, \hat{\Gamma}_{\hat{\tau}}}, P_{F, \hat{\Gamma}_{\hat{\tau}}}\right)$. Now we 
have

$$
\begin{aligned}
E_{D T}= & \lim _{N \rightarrow \infty}-\frac{1}{N} \ln \left(P_{1} P_{M}^{*}+P_{0} P_{F}^{*}\right) \\
\leq & \lim _{N \rightarrow \infty}-\frac{1}{N} \ln \min \left(P_{0}, P_{1}\right) \\
& +\lim _{N \rightarrow \infty}-\frac{1}{N} \ln \min \left(P_{M, \hat{\Gamma}_{\hat{\tau}}}, P_{F, \hat{\Gamma}_{\hat{\tau}}}\right) \\
= & E_{M, \hat{\Gamma}_{\hat{\tau}}}
\end{aligned}
$$

where the last equality is because $E_{F, \hat{\Gamma}_{\hat{\tau}}}=E_{M, \hat{\Gamma}_{\hat{\tau}}}$. Equation (58) suggests that $E_{D T}$ is less than or equal to the error exponent of a test with constant threshold $\hat{\tau}$. However, the optimal test should have the largest error exponent. As a result, we have $E_{D T}=E_{M, \hat{\Gamma}_{\hat{\tau}}}$. This finishes the proof.

\section{REFERENCES}

[1] Viswanathan, R. and Varshney, P. K Distributed detection with multiple sensors: Part I-Fundamentals. Proceedings of the IEEE, 85 (Jan. 1997), 54-63.

[2] Blum, R. S., Kassam, S. A., and Poor, H. V. Distributed detection with multiple sensors: Part II-Advanced topics.

Proceedings of the IEEE, 85 (Jan. 1997), 64-79.

[3] Srinivasan, $\mathrm{R}$. Designing distributed detection systems. IEE Proceedings-F, 140 (June 1993), 191-197.

[4] Thomopoulos, S. C. A. and Zhang, L. Distributed decision fusion with networking delays and channel errors. Information Sciences, 66 (Dec. 1992), 91-118.

[5] Lin, Y., Chen, B., and Varshney, P. K. Decision fusion rules in multi-hop wireless sensor networks.

IEEE Transactions on Aerospace and Electronic Systems, 41 (Apr. 2005), 475-488.

[6] Chen, B., et al.

Channel aware decision fusion in wireless sensor networks.

IEEE Transactions on Signal Processing, 52 (Dec. 2004), 3454-3458.

[7] Niu, R., Chen, B., and Varshney, P. K.

Fusion of decisions transmitted over Rayleigh fading channels in wireless sensor networks.

IEEE Transactions on Signal Processing, 54 (Mar. 2006), 1018-1027.

[8] Liu, B. and Chen, B.

Channel-optimized quantizers for decentralized detection in sensor networks.

IEEE Transactions on Information Theory, 52 (July 2006), 3349-3358.

[9] Chamberland, J. and Veeravalli, V. V.

Asymptotic results for decentralized detection in power constrained wireless sensor networks.

IEEE Journal on Selected Areas in Communications, 22 (Aug. 2004), 1007-1015.

[10] Jayaweera, S. K.

Bayesian fusion performance and system optimization for distributed stochastic Gaussian signal detection under communication constraints.

IEEE Transactions on Signal Processing, 55 (Apr. 2007), $1238-1250$
[11] Gini, F., Lombardini, F., and Verrazzani, L.

Decentralised detection strategies under communication constraints.

IEE Proceedings-Radar, Sonar and Navigation, 145 (1998), 199-208.

[12] Yu, C. and Varshney, P. K.

Bit allocation for discrete signal detection. IEEE Transactions on Communications, 46 (Feb. 1998), 173-175.

[13] Chamberland, J. and Veeravalli, V. V.

Decentralized detection in sensor networks. IEEE Transactions on Signal Processing, 51 (Feb. 2003), 407-416.

[14] Rago, C., Willett, P., and Bar-Shalom, Y.

Censoring sensors: A low-communication-rate scheme for distributed detection.

IEEE Transactions on Aerospace and Electric Systems, 32 (Apr. 1996), 554-568.

[15] Jiang, R. and Chen, B.

Fusion of censored decisions in wireless sensor networks. IEEE Transactions on Wireless Communications, 4 (Nov. 2005), 2668-2673.

[16] Appadwedula, S., Veeravalli, V. V., and Jones, D. L. Energy-efficient detection in sensor networks. IEEE Journal on Selected Areas in Communications, $\mathbf{2 3}$ (Apr. 2005), 693-702.

[17] Mergen, G., Naware, V., and Tong, L.

Asymptotic detection performance of type-based multiple access over multiaccess fading channels.

IEEE Transactions on Signal Processing, 55 (Mar. 2007), 1081-1092.

[18] Liu, K. and Sayeed, A. M.

Type-based decentralized detection in wireless sensor networks.

IEEE Transactions on Signal Processing, 55 (May 2007), $1899-1910$

[19] Li, W. and Dai, H.

Distributed detection in wireless sensor networks using a multiple access channel.

IEEE Transactions on Signal Processing, 55 (Mar. 2007), 822-833.

[20] Cover, T. and Thomas, J.

Elements of Information Theory (2nd ed.).

Hoboken, NJ: Wiley-Interscience, 2006.

[21] Anandkumar, A. and Tong, L.

Type-based random access for distributed detection over multiaccess fading channels.

IEEE Transactions on Signal Processing, 55 (Oct. 2007), 5032-5043.

[22] Liu, K., El Gamal, H., and Sayeed, A. Decentralized inference over multiple-access channels. IEEE Transactions on Signal Processing, 55 (July 2007), 3445-3455.

[23] Mergen, G., Sirkeci-Mergen, B., and Gastpar, M.

Sufficient-statistics based multiple access over wireless fading channel.

In Proceedings of GLOBECOM'08, New Orleans, LA, Dec. 2008, 1-5.

[24] Lin, Y., Chen, B., and Tong, L. Distributed detection over multiple access channels. In Proceedings of ICASSP'07, vol. 3, Honolulu, HI, Apr. 2007, 541-544.

[25] Laneman, J. N., Tse, D. N. C., and Wornell, G. W. Cooperative diversity in wireless networks: Efficient protocols and outage behavior.

IEEE Transactions on Information Theory, 50 (Dec. 2004), 3062-3080 
[26] Gastpar, M., Rimoldi, B., and Vetterli, M.

To code, or not to code: Lossy source-channel communication revisited.

IEEE Transactions on Information Theory, 49 (May 2003), $1147-1158$.

[27] Zacharias, L. and Sundaresan, R.

Decentralized sequential change detection using physical layer fusion.

IEEE Transactions on Wireless Communications, 7 (Dec. 2008), 4999-5008.

[28] Mudumbai, R., Barriac, G., and Madhow, U.

On the feasibility of distributed beamforming in wireless networks.

IEEE Transactions on Wireless Communications, 6 (May 2007), 1754-1763.

[29] Mudumbai, R., et al.

Distributed transmit beamforming: Challenges and recent progress.

IEEE Commununications Magazine, 47 (Feb. 2009), 102-110.
[30] Li, F. and Evans, J. S.

Decision fusion over noncoherent fading multiaccess channels.

In Proceedings of GLOBECOM'08, New Orleans, LA, Nov. 2008, 1-5.

[31] Dembo, A. and Zeitouni, O.

Large Deviations Techniques and Applications (2nd ed.). New York: Springer, 1998.

[32] Varshney, P. K.

Distributed Detection and Data Fusion. New York: Springer, 1996.

[33] Meserve, B. E.

Fundamental Concepts of Algebra.

Reading, MA: Addison-Wesley, 1953. 

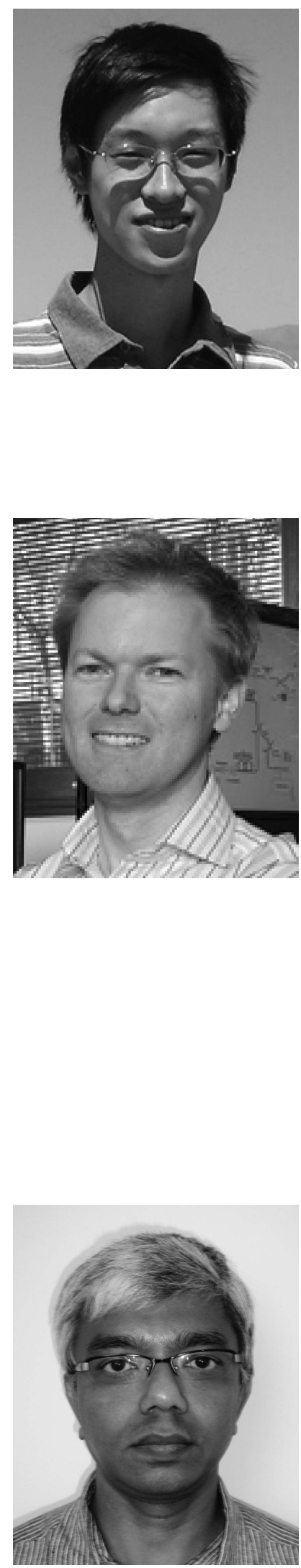

Feng Li (S'09) was born in Shanghai, China, in 1981. He received the B.E. degree from Shanghai Jiao Tong University, China, in 2004, and the Ph.D. degree from the Department of Electrical and Electronic Engineering, the University of Melbourne, Australia, in 2010.

He is currently a research fellow in the Department of Electrical and Electronic Engineering, the University of Melbourne. He served as a lecturer in the same department from 2009 to 2010 . He worked at NEC Australia from 2006 to 2007. His research interests include statistical signal processing, signal processing for wireless sensor networks, communication systems, and information theory.

Dr. Li received the best student paper award at the Australian Communications Theory Workshop twice (in 2008 and 2009). He served as the Local Chair of Australian Communications Theory Workshop in 2011.

Jamie Evans (M'98) was born in Newcastle, Australia, in 1970. He received the B.S. degree in physics and the B.E. degree in computer engineering from the University of Newcastle, Australia, in 1992 and 1993, respectively, where he received the University Medal upon graduation. He received the M.S. and the $\mathrm{Ph} . D$. degrees from the University of Melbourne, Australia, in 1996 and 1998, respectively, both in electrical engineering, and was awarded the Chancellor's Prize for excellence for his Ph.D. thesis.

From March 1998 to June 1999, he was a visiting researcher in the Department of Electrical Engineering and Computer Science, University of California, Berkeley. He returned to Australia to take up a position as lecturer at the University of Sydney, Australia, where he stayed until July 2001. Since that time, he has been with the Department of Electrical and Electronic Engineering, University of Melbourne, where he is now a professor. His research interests are in communications theory, information theory, and statistical signal processing with a focus on wireless communications networks.

Subhrakanti Dey (M'96-SM'06) was born in Calcutta, India, in 1968. He received the B.Tech. and M.Tech. degrees from the Department of Electronics and Electrical Communication Engineering, Indian Institute of Technology, Kharagpur, India, in 1991 and 1993, respectively, and the Ph.D. degree from the Department of Systems Engineering, Research School of Information Sciences and Engineering, Australian National University, Canberra, Australia, in 1996.

$\mathrm{He}$ has been with the Department of Electrical and Electronic Engineering, University of Melbourne, Parkville, Australia, since February 2000, where he is currently a full professor. From September 1995 to September 1997 and September 1998 to February 2000, he was a postdoctoral research fellow with the Department of Systems Engineering, Australian National University. From September 1997 to September 1998, he was a post-doctoral research associate with the Institute for Systems Research, University of Maryland, College Park. His current research interests include networked control systems, wireless communications and networks, signal processing for sensor networks, and stochastic and adaptive estimation and control.

Dr. Dey currently serves on the Editorial Board of the Elsevier Systems and Control Letters. He was also an associate editor for the IEEE Transactions on Automatic Control during 2005-2007 and an Associate Editor for the IEEE Transactions on Signal Processing during 2007-2010. He is a Senior Member of IEEE. 